\title{
Colloquium: Physical approaches to DNA sequencing and detection
}

\author{
Michael Zwolak ${ }^{*}$ \\ Physics Department, California Institute of Technology, Pasadena, California 91125, USA \\ and Theoretical Division MS-B213, Los Alamos National Laboratory, Los Alamos, \\ New Mexico 87545, USA \\ Massimiliano Di Ventra ${ }^{\dagger}$ \\ Department of Physics, University of California, San Diego, La Jolla, California 92093, \\ USA
}

(Published 2 January 2008)

\begin{abstract}
With the continued improvement of sequencing technologies, the prospect of genome-based medicine is now at the forefront of scientific research. To realize this potential, however, a revolutionary sequencing method is needed for the cost-effective and rapid interrogation of individual genomes. This capability is likely to be provided by a physical approach to probing DNA at the single-nucleotide level. This is in sharp contrast to current techniques and instruments that probe (through chemical elongation, electrophoresis, and optical detection) length differences and terminating bases of strands of DNA. Several physical approaches to DNA detection have the potential to deliver fast and low-cost sequencing. Central to these approaches is the concept of nanochannels or nanopores, which allow for the spatial confinement of DNA molecules. In addition to their possible impact in medicine and biology, the methods offer ideal test beds to study open scientific issues and challenges in the relatively unexplored area at the interface between solids, liquids, and biomolecules at the nanometer length scale. This Colloquium emphasizes the physics behind these methods and ideas, critically describes their advantages and drawbacks, and discusses future research opportunities in the field.
\end{abstract}

DOI: $10.1103 / \operatorname{RevModPhys.80.141}$

PACS number(s): 87.14.G-, 87.15.Cc, 87.15.H-

\section{CONTENTS}

I. Introduction

II. Current Sequencing Techniques

III. Physical Characteristics of DNA

A. Structure

B. Electronics

IV. Nanopores and Polynucleotides

A. Nanopore-polynucleotide concept

B. Nanopore characteristics

1. Biological pores

2. Synthetic pores

C. DNA translocation

1. Universal properties

2. Specifics

D. Pore and ion electronics

1. Ionic and blockade currents

2. Electrostatics

V. Sequencing and Detection

A. Electronic detection

1. Ionic blockade

2. Transverse electronic current

3. Capacitance

B. Optical detection

C. Force detection

VI. Conclusions

*mpz@lanl.gov

†diventra@physics.ucsd.edu

Acknowledgments

References

141

142

143

143

145

146

147

148

148

149

150

150

152

153

153

154

155

155

155

158

160

161

162

162
Since the first sequencing of the full human genome (Lander et al., 2001; Venter et al., 2001) and decades of sequencing improvements (Chan, 2005), genome-based medicine has come ever closer to reality. A low-cost and rapid method to sequence DNA would dramatically change the medical field, and would give us new tools to study biological functions and evolution.

This new technology would allow us to sequence and compare a plethora of individual human genomes, enabling us to locate sequences that cause hereditary diseases and to discover mutated sequences. Individual medical consumers can then be tested for these known genetic defects, and medicine and treatments can be tailored to their specific condition(s). This latter goal is known as personalized medicine and would be a tremendous advancement in the medical field. Furthermore, the genomic information gathered could be used in the study of biology by, for example, comparing genomes between species to find common properties and functions. This information could be used both medically (e.g., in animal testing of potential cures for humans) and to examine the evolutionary heritage of all species.

In order to reach the goal of a rapid and low-cost sequencing method, one cannot rely only on current techniques, which involve costs of about 10 million USD and several months time to sequence a single human 
genome (Fredlake et al., 2006). Improvements of current technology face both fundamental and practical limitations, such as a small-on the order of 1000 basesread-length limit using electrophoresis (Chan, 2005), which will restrict the impact of subsequent developments in this direction. Therefore, radically novel ideas need to be implemented and demonstrated to be cost effective and accurate.

There are several candidates that may fill this role (Kasianowicz et al., 1996, 2001; Akeson et al., 1999; Deamer and Akeson, 2000; Deamer and Branton, 2002; Heng, Aksimentiev, Ho, Dimitrov, et al., 2005; Zwolak and Di Ventra, 2005; Gracheva, Xiong, Aksimentiev, et al., 2006; Lagerqvist et al., 2006; NHGRI, 2006; Lee and Meller, 2007; Lindsay et al., 2007). All these candidates have one trait in common: they employ nanoscale probes to examine the structural or electronic signatures of individual DNA bases. That is, they rely on physical differences between the bases. This is a major departure from existing sequencing paradigms that rely on chemical techniques and physical differences between strands of DNA.

Most importantly, these proposals challenge our understanding of, and ability to manipulate and probe, physical processes at the interface between solids, liquids, and biomolecules down to the nanometer-scale regime (Di Ventra et al., 2004). Indeed, in order to understand the feasibility, speed, and accuracy of these novel approaches, we are naturally led to examine several physical questions about the individual bases and the influence of the solid/liquid environment:

- What is the difference in magnitude of physically measurable properties between the bases?

- How do the nucleotide structural dynamics affect the measurable signals?

- How do the different bases interact with the components of the detection apparatus, e.g., the nanopore, the surfaces, the electrodes, a scanning probe tip, or the other molecules present?

- How does the atomic makeup and structure of the different bases affect the surrounding fluid and ionic dynamics? And conversely, how do the latter affect the structure and electronics of the bases?

- What are the significant sources of noise?

In addition, many of the suggested sequencing methods rely on nanopores either as a housing for the nanoscale probe(s) or as a restriction that causes differentiation in some signal between the bases. Thus, the fabrication of the nanopores and the DNA translocation dynamics have an important bearing on the following questions:

- How well can one probe the DNA on the single-base scale according to the dimensions and "uniformity" of nanopores?

- How fast can one probe base differences?

- What are the limits on read lengths?
- How fast and regular does the DNA translocate through the pore?

The above points beget even more basic and general physical questions:

- What is the meaning and role of electronic screening at the nanometer scale and in a strongly confined and fluctuating environment?

- What is the meaning of capacitance, thermal energy, charging energy, etc., under these atypical conditions and at small length scales? How do these quantities evolve into their corresponding bulk properties?

- Do liquids show unexpected dynamical features at the nanoscale?

- How do electrons move in "soft" materials and dynamical environments?

These questions will accompany us throughout this Colloquium. We stress their importance for the detection and sequencing approaches, review some partial answers found in the existing literature, and point out possible future research directions to explore them in more depth.

The Colloquium is organized as follows. In Sec. II, we give a very brief account of current sequencing techniques. This primer will help the reader become familiar with the state of the art in this field. In Sec. III, we outline the physical properties of DNA and its bases. In Sec. IV, we discuss nanopores as a useful building block for rapid DNA sequencing and detection. After this, we move on to physical approaches to DNA sequencing in Sec. V. Finally, we conclude in Sec. VI.

\section{CURRENT SEQUENCING TECHNIQUES}

A thorough introduction to existing sequencing methods can be found in Dovichi and Zhang (2000), JGI (2004), Chan (2005), and Fredlake et al. (2006), and references therein provide a more technical account. Present-day sequencing methods are an improved version of the Sanger method (Sanger et al., 1977). The sequencing process can be divided into four overall steps (Chan, 2005): (i) DNA isolation, (ii) sample preparation, (iii) sequence production, and (iv) assembly and analysis. Step (i) is simply the isolation of the strand of DNA that needs to be sequenced. Within step (ii), the DNA needs to be replicated and also broken into (many) very short strands. The length of the strands is dictated by the actual sequencing technology used.

Step (iii) combines three components for the detection of the bases in the DNA sequence, as shown in Fig. 1. First, chemical elongation creates labeled strands of DNA with the random insertion of a chain-terminating nucleotide (introduced by Sanger et al., 1977). Second, an electrophoretic ${ }^{1}$ process spatially separates the differ-

\footnotetext{
${ }^{1}$ Electrophoresis is a general term meaning the action of driving charged molecules or particles in a solution with an electric
} 


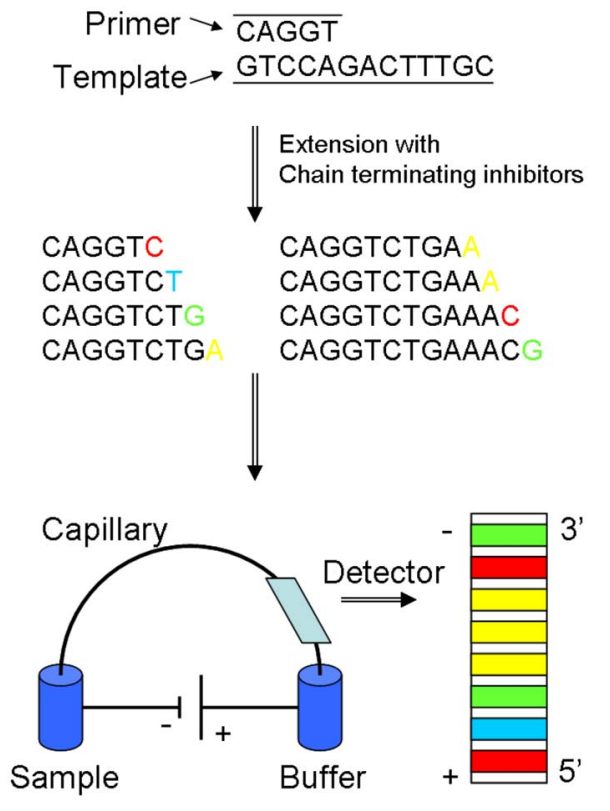

FIG. 1. (Color online) The three processes used to produce the sequence of a strand of DNA. One starts with a DNA strand to be sequenced, called the template, to which one adds a primer that is complementary to part of the template. A DNA polymerase reaction extends the sequence starting from the primer by adding nucleotides to the $3^{\prime}$ end. In the presence of a smaller amount of dideoxynucleotides, the chains terminate at various places along the complementary strand, and after denaturation, produce single strands of different lengths. These strands are then sorted with capillary electrophoresis and detected by laser excitation of fluorescent tags (one can either use multiple lanes each with its own dideoxynucleotide and fluorescently tagged primer or use fluorescently tagged dideoxynucleotides). The detection information is then sent to a computer for assembly and postprocessing.

ent lengths of DNA in a porous matrix. Third, an optical readout detects the fluorescent end groups or primers, which indicates the last base on each of the different lengths of DNA.

Step (iv) is the postprocessing of the sequence data, which involves the reassembly of the short sequences to get the complete sequence of the original strand of DNA. Because of this, the short strands of DNA are required to have large overlapping sequences in order to match them up.

From the above discussion, one sees that the current methods rely on very complex sample preparation and postprocessing of the data. One of the main causes of this complexity is a fundamental barrier to the maximum read length achievable when using electrophoresis (Chan, 2005). ${ }^{2}$ The read length is the longest strand of DNA that can be sequenced accurately and efficiently

field. More specifically, it is taken to mean driving the particles through a porous matrix.

${ }^{2}$ Practical application of the chain-terminating chemistry can also limit read lengths. within step (iii). The read length is limited because electrophoresis is sensitive to the physical difference between different lengths of (single-stranded) DNA. Thus, intuitively, one expects that, as the strand gets longer, distinguishing a strand of $N$ bases from one of $N+1$ bases becomes increasingly difficult, because the percentage difference in the strand properties tends to zero. As a consequence, if a sequencing scheme allows for longer read lengths of the DNA, this simplifies sample preparation and postprocessing. Since the physical schemes described in this Colloquium rely on singlenucleotide detection, the amount of sample preparation should be considerably reduced. The electrophoresis step is also intrinsically slow (Chan, 2005).

This Colloquium focuses on methods that improve and modify step (iii) of the sequencing process. However, as we just saw in the case of the read length, the four sequencing steps are not independent. Modifications to step (iii) can reduce the time and complexity of steps (ii) and (iv) and correspondingly reduce their costs. Thus, the technological motivation for improving step (iii) comes from its pivotal role in the sequencing process.

\section{PHYSICAL CHARACTERISTICS OF DNA}

In this section, we give an introduction to the basic properties and structure of the polynucleotides ${ }^{3}$ (PNs), and their constituents. The bases are very similar to each other and thus these properties are crucial to understanding their distinguishability via size, electronic states, or interactions with the surroundings.

\section{A. Structure}

Both deoxyribonucleic acid (DNA) and ribonucleic acid (RNA) are built up of different bases attached to a sugar-phosphate backbone. The five bases that make up these polymers are shown in Fig. 2. These can be classified into two categories: the purine bases (A and $\mathrm{G}$ ) and the pyrimidine bases $(\mathrm{C}, \mathrm{T}$, and $\mathrm{U})$. The purine bases consist of a six- and a five-membered ring with a common edge. The pyrimidines have just a six-membered ring. These classifications are natural and based on the chemical structure. We will see that, for many physical properties, the distinction into purines and pyrimidines is not helpful. However, one would expect that some physical properties would divide along this classification. For instance, purines are larger and thus one might expect that they would interact more strongly with surfaces in a confined space.

The backbone structure of a polynucleotide is shown in Fig. 3(a). Each monomer unit is a nucleotide, which consists of a base, phosphate group $\left(\mathrm{PO}_{4}\right)$, and sugar. For DNA (RNA), the latter is the deoxyribose (ribose) sugar shown in Fig. 3(b) and 3(c). The difference of only

\footnotetext{
${ }^{3}$ One also refers commonly to oligonucleotides, indicating a short strand of DNA or RNA.
} 


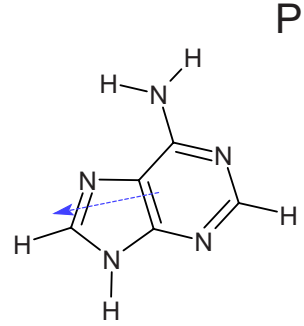

Adenine
Purine

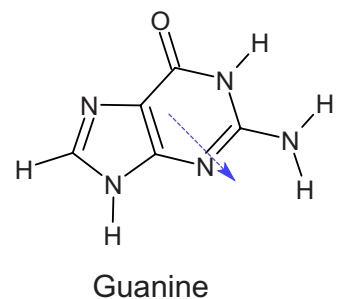

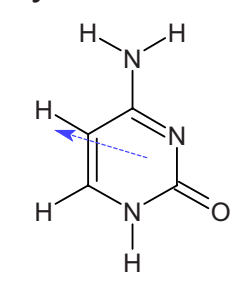

Cytosine<smiles>CCOc1cccc(=O)[nH]1</smiles>

Uracil
FIG. 2. (Color online) Atomistic structure of the nitrogenous bases found in DNA and RNA. For DNA, these are the purine bases adenine $(\mathrm{A})$ and guanine $(\mathrm{G})$, and the pyrimidine bases thymine $(\mathrm{T})$ and cytosine $(\mathrm{C})$. For most ribonucleic acids, uracil (U) takes the place of thymine. Each base is shown so that the bottom-most hydrogen indicates where the base attaches to the sugar group of the backbone. Also shown are the directions of their dipole moments.

a single hydroxyl group $(\mathrm{OH})$ between RNA and DNA can be important for the global structure of the polynucleotide. Within the repeat unit of the polynucleotides, there is one phosphate group, i.e., a nucleotide monophosphate. However, triphosphate monomers are used in chain extension reactions. The formation of the double-stranded (ds) DNA helix occurs by the WatsonCrick base pairing, where A pairs with $T$ and $G$ pairs with $\mathrm{C}$.

One of the most important properties of polynucleotides is that they are charged in solution. The $p K_{a}$ of the phosphate group, i.e., the measure of how readily that group will give up a hydrogen cation (proton), is near 1 . Thus, under most ionic conditions (including physiological $p \mathrm{H}$ ), the backbone will contain a single negative charge for each nucleotide unit (or two negative charges for a Watson-Crick pair of nucleotides in a double strand). In solution, though, there will be, on average, nearby counterions such as sodium $\left(\mathrm{Na}^{+}\right)$, potassium $\left(\mathrm{K}^{+}\right)$, or magnesium $\left(\mathrm{Mg}^{2+}\right)$, which neutralize a part of this charge. It is because the strand of nucleotides is charged that one can pull the DNA through nanopores with an electric field.

There are other important properties that will help us to understand the experiments and theoretical proposals below. For instance, a polynucleotide has a global orientation, with one end a $5^{\prime}$ and the other end a $3^{\prime}$, as described in Fig. 3. We show in Sec. IV that this is important for the structural dynamics of polynucleotides translocating through a nanopore.
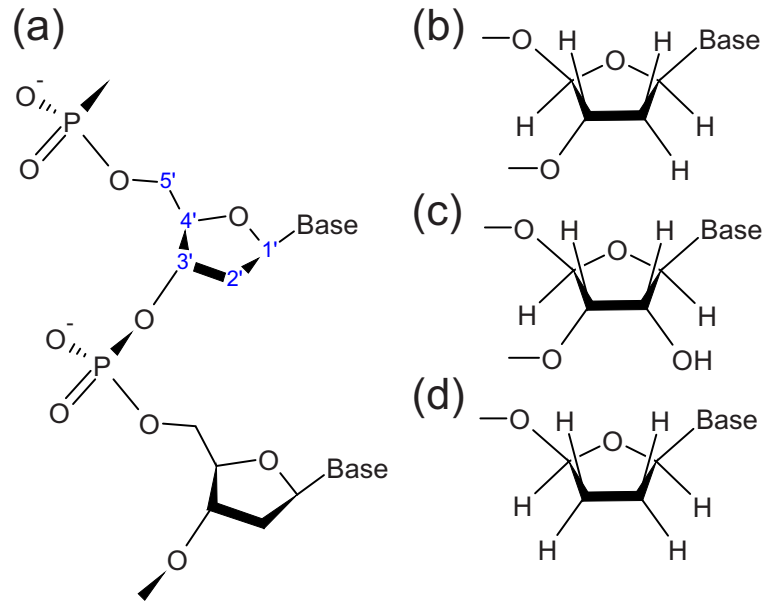

(c)

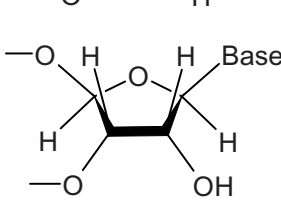

(d)

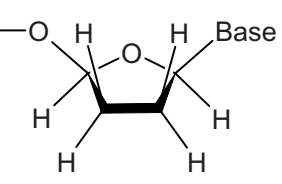

FIG. 3. (Color online) The atomistic structure of the nucleic acid backbones. (a) Two repeat units of the deoxyribonucleic acid backbone. The phosphate groups have a negative charge in solution, which is shared between two oxygens solely bound to the phosphorus atom. Also shown is the numbering system. The sugar carbon attached to the base is labeled 1' (the prime indicating that the numbering is on the sugar), then the other carbons are labeled around the ring up to $5^{\prime}$. This is the origin of the terminology $3^{\prime}$ and $5^{\prime}$. A single strand can end after the $3^{\prime}$ carbon or the $5^{\prime}$ carbon. The atomistic structure, as a unit in a polynucleotide, of the (b) deoxyribose sugar, (c) ribose sugar, and (d) dideoxyribose sugar. The latter is used in the Sanger method (see Sec. II), because the absence of the 3' hydroxyl group will not allow further chain extension. The typical nomenclature is to add a "d" in front of the base to indicate that it is attached to a deoxyribose sugar instead of a ribose sugar, e.g., poly (dA) is a polynucleotide of DNA, while poly (A) is a polynucleotide of RNA.

There is also a global property called secondary structure. For ds-DNA, for instance, there exist several different types of helices. The most common ones are called A-DNA and B-DNA. What changes the global structure of DNA between A- and B-DNA is the ionic and water environment. B-DNA is preferred in an aqueous environment because water molecules can bind in the grooves along the helix. The base-pair-base-pair distance in the B-DNA helix is $3.4 \AA$ and there is a $36^{\circ}$ angle between them, which gives about 10 bases per turn of the helix. The diameter of B-DNA is $\sim 2 \mathrm{~nm}$. A-DNA has different physical dimensions. The difference between B-DNA and A-DNA is probably not important for sequencing, but some proposals for detection of dsDNA will be affected by such a change in global structure due to the environmental conditions. Furthermore, there is a process called denaturation in which the two strands in ds-DNA unbind into single-stranded (ss) DNA molecules.

Single strands show secondary structure as well, which also depends on ionic conditions and temperature. A schematic of secondary structure in ss-RNA is shown in Fig. 4. Generally, one can think of secondary structure as a result of the competition between enthalpic and entropic factors. The interaction energy of base stacking is 


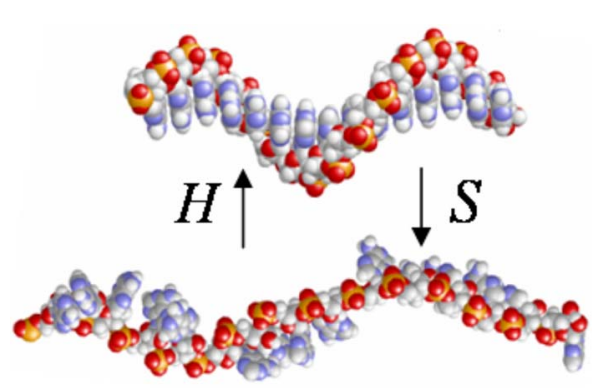

FIG. 4. (Color online) Schematic of a secondary structure of ss-RNA. On the top, stacked bases forming a single-stranded helix due to enthalpic effects (denoted as $H$ ). Entropic effects (denoted by $S$ ) transform this structure into the one on the bottom, a random coil polymer. The homogeneity of the strand is important for secondary structure, as are the ionic conditions and the temperature. For instance, $\operatorname{poly}(\mathrm{C})$ at room temperature and neutral $p \mathrm{H}$ is mostly a single-stranded helix $1.3 \mathrm{~nm}$ in diameter (Arnott et al., 1976).

the main factor favoring secondary structure (Searle and Williams, 1993), such as the helix in Fig. 4. On the other hand, the entropic factors are mainly due to rotations of the backbone degrees of freedom (Searle and Williams, 1993). If one raises the temperature, entropic factors will dominate and bring the strand to a random coil form. When this transition happens depends on the bases in the strand (Vesnaver and Breslauer, 1991), ionic conditions (Dewey and Turner, 1979), and other environmental factors (Freier et al., 1981). The effect of secondary structure has already been seen in the ionic currents through nanopores (Akeson et al., 1999; Meller et al., 2000).

Another type of secondary structure is a hairpin. RNA and DNA hairpins are single strands of RNA and DNA that wrap around to form a double strand. However, in doing so, they have to form a loop of unpaired bases, which causes an unfavorable strain on their formation. The double-stranded portion is called the stem, and sometimes a single-stranded portion can exist on the chain as well. This latter type of hairpin is used in some of the experiments described below.

We also add that in some of the proposals for sequencing, an exonuclease could be used to chop the DNA up into individual nucleotides. A nuclease is an enzyme that acts as a catalyst to polynucleotide breakup, e.g., the linking oxygen between nucleotide repeat units can be hydrolyzed to two $\mathrm{OH}$ groups. An exonuclease is a nuclease that "eats away" at the ends of the polynucleotide, breaking off one nucleotide at a time.

Some particular properties of interest to us are the base and nucleotide sizes. The sizes of the DNA bases and corresponding deoxyribonucleic acids are shown in Table I. Using geometries from Zwolak and Di Ventra (2005), these sizes were computed by drawing a sphere around each atom of its van der Waals radius, and taking the union of these spheres. From the table, one can observe that, even in the most ideal case, the size of the
TABLE I. Sizes of the DNA bases $\left(V_{B}\right)$ and nucleotides $\left(V_{N}\right)$ in $\AA^{3}$. Also given are the surface areas $A_{B}$ and $A_{N}$ in $\AA^{2}$. We define the fraction of free volume left $F_{d}=\left(V_{p}-V_{N}\right) / V_{p}$, where the pore volume $V_{p}=\pi l d^{2} / 4$ is for a cylinder of length $l$ and diameter $d$. Each nucleotide is assumed to occupy a length $l$ $\approx 7 \AA$, which is its own length in a completely extended strand. We consider pore diameters of 15 and $20 \AA$, which are approximately equal to the $\alpha$-hemolysin pore diameter in its stem. For comparison, the backbone and $U$ volume (surface area) are 214 (225) and 128 (142), respectively.

\begin{tabular}{llll}
\hline \hline Base & $V_{B}\left(A_{B}\right)$ & $V_{N}\left(A_{N}\right)$ & $F_{15}\left(F_{20}\right)$ \\
\hline $\mathrm{A}$ & $157(166)$ & $349(340)$ & $0.72(0.841)$ \\
$\mathrm{G}$ & $168(177)$ & $359(351)$ & $0.71(0.837)$ \\
$\mathrm{C}$ & $133(147)$ & $324(319)$ & $0.74(0.853)$ \\
$\mathrm{T}$ & $150(163)$ & $339(331)$ & $0.73(0.846)$ \\
\hline \hline
\end{tabular}

base alone is unlikely to provide a distinguishable signal. This will be discussed in Sec. IV.D.

\section{B. Electronics}

Two detection and sequencing schemes propose to use transport and voltage fluctuations to distinguish the bases. These will be sensitive to the electronic structure of the different nucleotides and also to the geometry and local environment.

Transport through nucleotides will detect differences in electronic states via their energy and spatial extension. To understand the differences between the bases, we compute the density of states of the nucleotides (placed between two electrodes) projected onto the backbone and bases, as shown in Fig. 5. The plot shows that the different nucleotides do not have a considerably different electronic structure, having molecular states very near in energy compared to the position of the Fermi level. Most of the density of states around the Fermi level is contributed by the backbone. This is due in part to the upright geometry in Fig. 5(b), which allows for a large contact area of the backbone with an electrode. The different spatial extension of the bases causes their contribution to the density of states (DOS) at the Fermi level to differ. These differences will be heavily influenced by the nucleotide geometry and orientation.

However, the basic input determining the baseelectrode coupling is the character of the molecular states. For the isolated bases, this is shown in Fig. 6 for the highest occupied molecular orbital (HOMO) and lowest unoccupied molecular orbital (LUMO). For nucleotides only weakly contacted with electrodes, the character of these states is going to determine how well the nucleotide can couple to charge carriers in the electrodes. We can see that, for all the bases, the states are distributed around the rings. These states remain that way even in the presence of the passivated or charged backbone (although they may not remain the HOMO and LUMO states of the nucleotide). The spatial extension of the base is roughly correlated with the relevant 


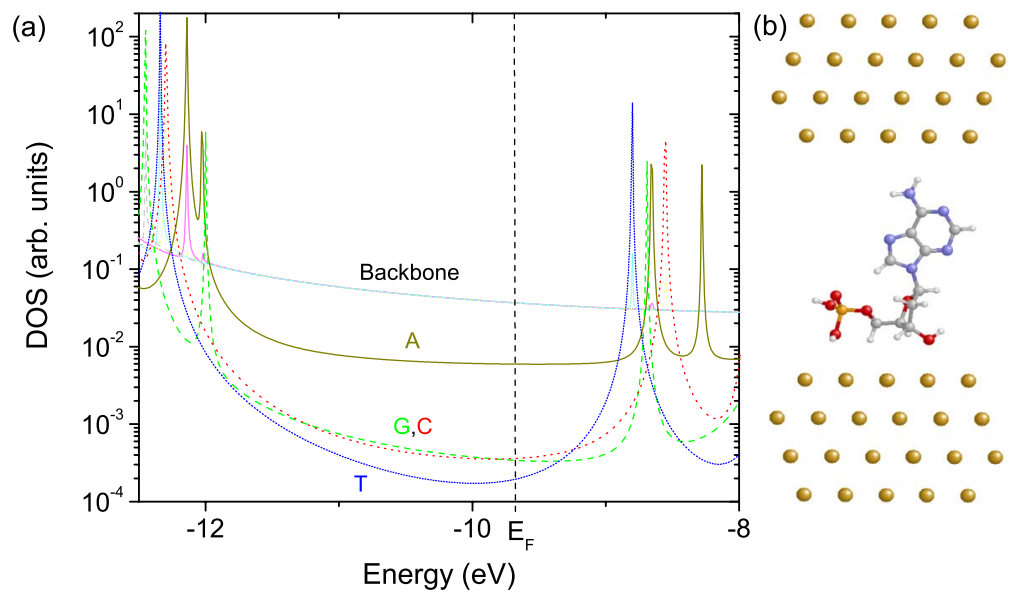

FIG. 5. (Color online) Electronic structure of the DNA nucleotides. (a) Projected density of states of the DNA nucleotides between two gold electrodes shown in (b), as computed by Zwolak and Di Ventra (2005) with tightbinding parameters. $E_{F}$ is the Fermi level of gold within the same approach.

electronic states and thus will determine in large part the coupling of the nucleotide to the electrodes.

On the other hand, other electronic approaches may measure the dipole and higher moments. The dipole moment magnitudes are shown in Table II, with the corresponding directions shown in Fig. 2. The dipole moments are for the isolated DNA bases and for the corresponding nucleotides. The moments and molecular wave functions were computed within the Hartree-Fock approximation using the geometries by Zwolak and Di Ventra (2005).

Finally, an important property of PNs relevant to the studies discussed in this Colloquium relates to their

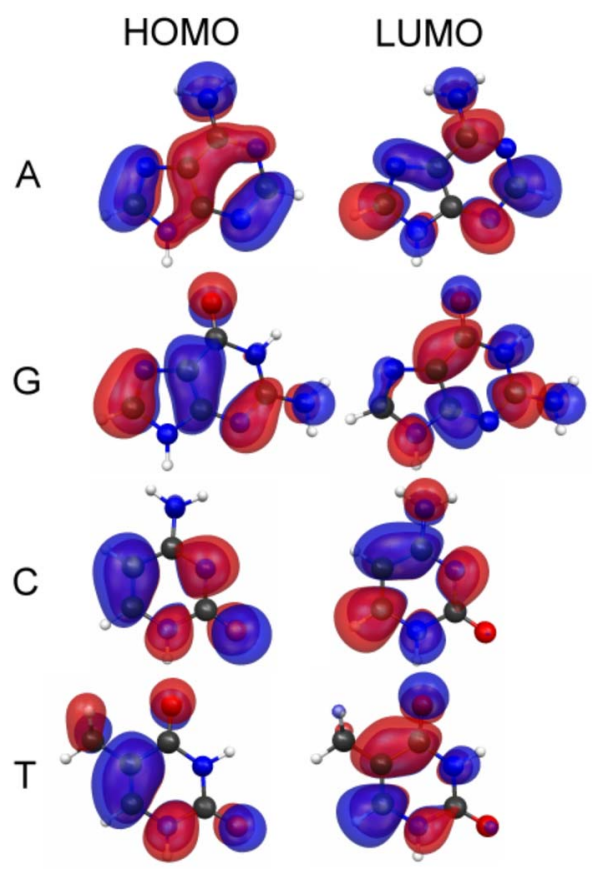

FIG. 6. (Color online) Isosurfaces of the HOMO and LUMO states of the isolated bases. The shades of gray (colors) indicate opposite signs of the wave function. The HOMO and LUMO states do not change from the individual base to the passivated nucleotide. However, when the backbone becomes charged in solution, the HOMO and LUMO states will shift in both character and energy. bonding properties at surfaces, and in particular at the interior surfaces of nanopores. These interactions, and their effect on the PN dynamics in confined geometries, are a property of both the nucleotides and the type of surface, thus making it a complex issue. We defer its discussion to later sections on particular experiments.

\section{NANOPORES AND POLYNUCLEOTIDES}

About a decade ago, Kasianowicz and collaborators were able to pull single-stranded polynucleotides through a biological nanopore by applying a voltage across the pore that pulls on the charged PN backbone (Kasianowicz et al., 1996). These authors detected the translocation of the PN via measurement of the blockade current, discussed below. Since this pioneering experiment, nanopores have been used to extract a variety of information characterizing the translocation, dynamics, and interactions of both single- and double-stranded PNs in nanopores (Kasianowicz et al., 1996, 2001; Akeson et al., 1999; Henrickson et al., 2000; Meller et al., 2000, 2001; Deamer and Branton, 2002; Chang et al., 2004; Chen et al., 2004; Fologea, Gershow, Ledden, et al., 2005; Mathé et al., 2005; Storm, Storm, Chen, et al., 2005; Butler et al., 2006). Further progress is being made by moving to synthetic nanopores where techniques are being developed to control the nanopore shapes, sizes, and other characteristics (Li et al., 2001, 2003; Storm et al.,

TABLE II. Theoretically calculated dipole moments of the bases $\left(p_{B}\right)$ and the deoxyribonucleic acids $\left(p_{N}\right)$ in debye $(\sim 0.21 \mathrm{e} \AA)$. For comparison, the dipole moment of $\mathrm{U}$ is $4.79 \mathrm{D}\left(p_{\mathrm{U}}^{\mathrm{expt}}=4.2 \mathrm{D}\right)$ and the backbone dipole by itself is 1.97 D. Experimental values $p_{B}^{\text {expt }}$ are from Kulakows et al. (1974), Weber and Craven (1990), and Devoe and Tinoco (1962).

\begin{tabular}{lccc}
\hline \hline Base & $p_{B}$ & $p_{B}^{\text {expt }}$ & $p_{N}$ \\
\hline $\mathrm{A}$ & 2.33 & 2.5 & 4.76 \\
$\mathrm{G}$ & 7.17 & 7.1 & 7.76 \\
$\mathrm{C}$ & 7.22 & 7.0 & 8.55 \\
$\mathrm{~T}$ & 4.72 & 4.1 & 7.56 \\
\hline \hline
\end{tabular}


2003; Wanunu and Meller, 2007). Successful sequencing and detection will require control of these characteristics.

In addition to experiments, theoretical and computational work is under way to help understand polynucleotide translocation through nanoscale pores. There are two fruitful approaches to this complex issue: phenomenological models and molecular dynamics. The phenomenological models provide a highly reduced description of the polymer dynamics, but they are able to elucidate the dependence of the dynamics on parameters such as the polymer length, pore dimensions, and applied field (Sung and Park, 1996; Lubensky and Nelson, 1999; Muthukumar, 1999, 2001; Chern et al., 2001; Chuang et al., 2001; Ambjörnsson et al., 2002; Kong and Muthukumar, 2002; Loebl et al., 2003; Meller, 2003; Slonkina and Kolomeisky, 2003; Luo et al., 2006; Matysiak et al., 2006; Tsai and Chen, 2007). On the other hand, if one wants to understand how to probe physical differences between the bases, an atomistic description of the polynucleotide dynamics is necessary. Moleculardynamics simulations coupled to other computational methods have been used in this context to study the signals and fluctuations expected when measuring different physical quantities as the PN translocates through the pore (Aksimentiev et al., 2004; Cui, 2004; Heng, Aksimentiev, Ho, Dimitrov, et al., 2005; Heng, Aksimentiev, Ho, Marks, et al., 2005; Jenkins et al., 2005; Gracheva, Aksimentiev, and Leburton, 2006; Gracheva, Xiong, Aksimentiev, et al., 2006; Heng et al., 2006, 2004; Lagerqvist et al., 2006, 2007a, 2007b; Muthukumar and Kong, 2006).

In this section, we first review the basic concepts of the nanopore-polynucleotide experiment. We then discuss some experimental results on biological and synthetic pores. We also include a number of interesting physical results on PN translocation and pore electronics, which demonstrate the wealth of fundamental physics that is contained in this new field.

\section{A. Nanopore-polynucleotide concept}

Prior to Kasianowicz et al. (1996), researchers were already interested in the ability to pull small charged molecules and polymers through ion channels (Henry et al., 1989; Bezrukov and Kasianowicz, 1993; Bayley, 1994; Bustamante et al., 1995; Kasianowicz and Bezrukov, 1995; Bezrukov et al., 1994, 1996). Thus, the basic question was whether PNs could also translocate through, and be detected by, a pore. A schematic of this process is shown in Fig. 7. The figure shows a membrane or thin layer that divides a solution into two half. An electrode is placed into each of the halves. Without the PN, electrodes pull ions through the channel to create an ionic current $I_{o}$ in the open channel (see Fig. 8).

Due to the negative charges on the phosphate groups, the PN is pulled to the positively biased (cathode) half of the solution. Eventually the strand is captured and enters the pore. During a time $t_{d}$, the translocation duration, the strand partially blocks ions from the pore as shown in Fig. 7(b). Even though the nucleotide is

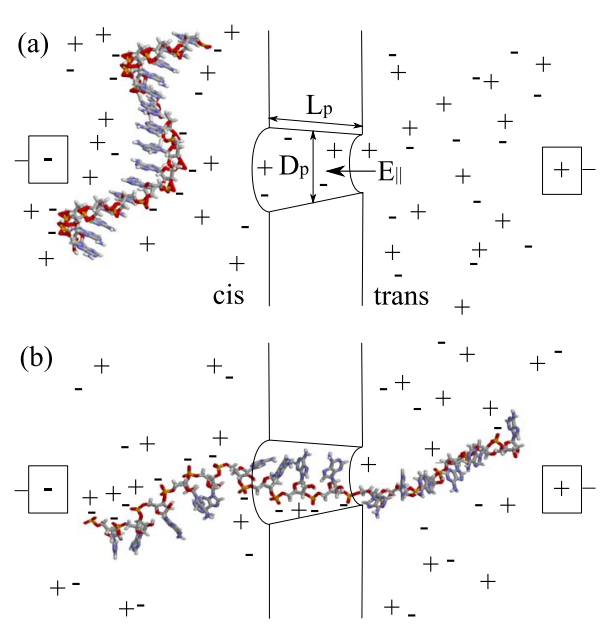

FIG. 7. (Color online) Schematic of the nanopore-PN experiment. A membrane divides the solution into what are commonly called the cis and trans chambers. (a) A bias across the separation creates a field $E_{\|}$across the membrane and drives an ionic current through the pore. (b) The field also pulls on the negatively charged PN backbone, which causes the PN to be captured by and then translocate through the pore. While the $\mathrm{PN}$ is within the pore, ions are partially prevented from occupying and flowing through the pore, thus reducing the ionic current. The pore is characterized by its length $L_{p}$ and average diameter $D_{p}$.

charged, it carries very little ionic current through the pore because of its slow velocity compared to the ions. Thus, its presence gives the blockade current $I_{b}$ shown in Fig. 8, so that the PN translocation event can be de-

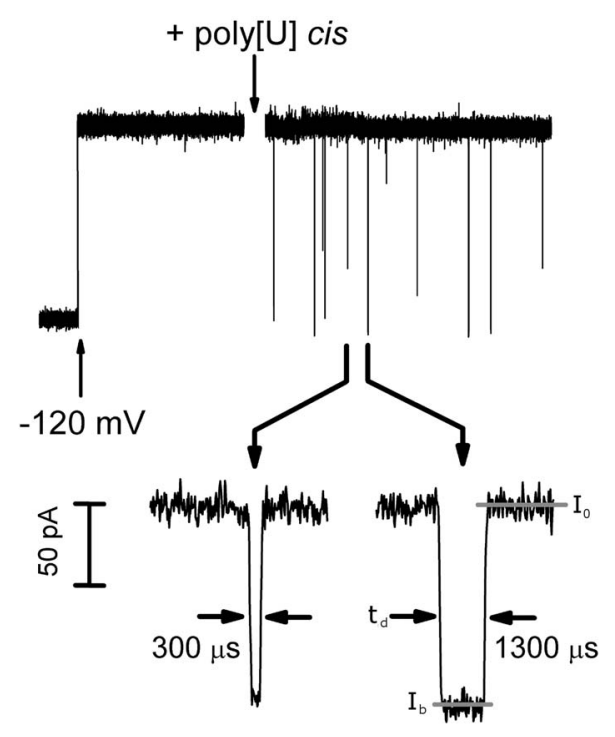

FIG. 8. Example of an ionic current signal. Upper: A voltage of $120 \mathrm{mV}$ drives a current through an $\alpha$-hemolysin pore. When poly (U) is added to the cis chamber, it generates blockade events. Lower: Two examples of blockade events. The average open pore current $I_{o}$, the average blockade current $I_{b}$, and the translocation duration $t_{d}$ are all indicated. Adapted from Kasianowicz et al., 1996. 
tected. The translocation events also provide a way to obtain information about the PN, such as its length, and limited information on composition and dynamics.

The actual values of the ionic current of the open pore and the blockade current result from several factors. The open pore resistance is determined by both an energy penalty (mainly electrostatic) and an entropic barrier to bringing charges into the pore. We show in Sec. IV.D that this is quite a complicated problem in itself, due to the microscopic details of electric fields in reduced geometries and fluctuating environments (e.g., the value of a screened charge in an electric field at the nanoscopic level cannot be simply viewed as a charge within a dielectric environment), and also the surface physics of pores in solution. The blockade current is also a complex phenomenon. We discuss this issue in the context of the particular experiments described below.

\section{B. Nanopore characteristics}

The current state of the art is divided into two lines of research. One line uses biological pores, generally $\alpha$-hemolysin pores (Song et al., 1996). These pores have the right size scale to detect differences in strands of PN. However, tailoring their characteristics, such as the pore diameter, is not straightforward. The other line of research operates with synthetic pores, which can be reasonably controlled down to the subnanometer range. One can also imagine making hybrid devices to help control pore properties.

The characteristics of the nanopore and the ability to control them will be extremely important for sequencing methods. For instance, methods that propose the use of nanoscale probes embedded in the pore will require a pore size that maximizes the signal difference between the bases beyond the many unavoidable sources of noise. In most cases, this means a pore diameter with the same width as a single strand of DNA. However, the maximization of the signal difference has to be balanced with other effects such as DNA-surface interaction, which is minimal with a large-diameter pore, and DNA capture and translocation, which will not occur at small pore sizes. In addition, the pore has to be fabricated in a way that makes possible the embedding of a nanoscale probe. This will put restrictions on the types of material suitable for the pore and its size and shape.

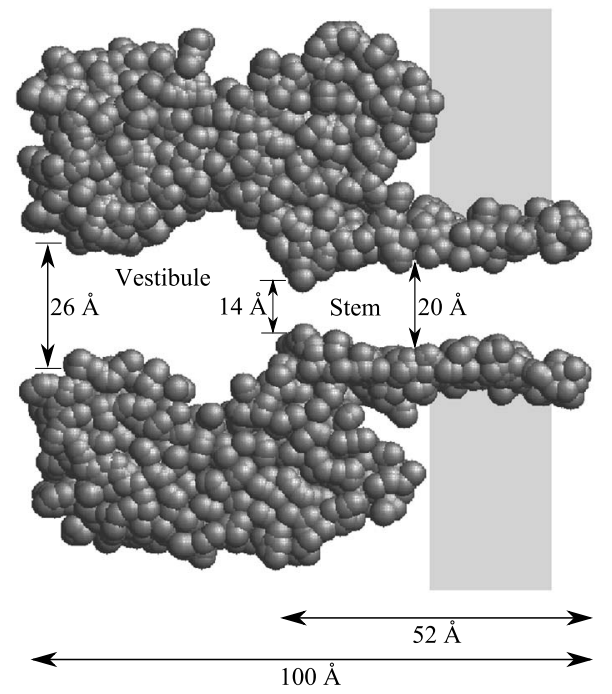

FIG. 9. Slab view of the $\alpha$-hemolysin pore. The uniform light gray area represents the lipid bilayer in which the $\alpha$-hemolysin assembles to make the pore. The initial part of the pore with a much wider diameter is the vestibule. The approximately 5-nm-long neck is the pore stem. Some other characteristics of the pore are an $\sim 18 \mathrm{~nm}^{3}$ stem volume (Deamer and Branton, 2002), and at $120 \mathrm{mV}$, the pore current is $120 \mathrm{pA}$, giving almost $10^{9}$ ions $/ \mathrm{s}$.

\section{Biological pores}

The $\alpha$-hemolysin pore is shown in Fig. 9. In this biological pore, the smallest restriction that the $\mathrm{PN}$ has to translocate through is $\sim 1.4 \mathrm{~nm}$. Thus, ds-DNA, at a diameter of $2 \mathrm{~nm}$, cannot translocate through the pore, but ss-DNA can (Kasianowicz et al., 1996). The pore is also both small and long enough that the PN has to be locally extended, which gives an entropic barrier for transport due to unraveling of the polymer. We give an overview of some $\alpha$-hemolysin experiments in Table III.

A typical experimental setup with an $\alpha$-hemolysin pore is shown in Fig. 10. There are two chambers, the cis and the trans, with a buffered solution, e.g., of $\mathrm{KCl}$. Between the two is a Teflon partition with a small orifice where the lipid bilayer is formed. When the hemolysin subunits are added, they spontaneously form into the pore. The formation of the pore is detected by the appearance of an ionic current between the two chambers.

TABLE III. Overview of some $\alpha$-hemolysin experiments. $T$ represents the temperature and $v_{\text {DNA }}$ is the DNA velocity through the pore. $\mathrm{p}(X)$ stands for a polymer of $X$, and $L$ is the length of the polymer in nucleotides (nt). The designation $3^{\prime} \leftrightarrow 5^{\prime}$ indicates an experiment that looked at the translocation direction.

\begin{tabular}{lccc}
\hline \hline Reference & PN & $L$ (nt) & Result \\
\hline Kasianowicz et al. $(1996)$ & $\mathrm{p}(\mathrm{U})$, others & $\sim 150-450$ & $t_{d} \propto L$ \\
Akeson et al. $(1999)$ & $\mathrm{p}(\mathrm{A}), \mathrm{p}(\mathrm{C}), \mathrm{p}(\mathrm{dC}), \mathrm{p}(\mathrm{U}), \mathrm{p}(\mathrm{A})(\mathrm{C})$ & $\sim 100-200$ & Discrimination \\
Meller et al. $(2000)$ & $\mathrm{p}(\mathrm{dA}), \mathrm{p}(\mathrm{dC}), \mathrm{p}(\mathrm{dA})(\mathrm{dC}), \mathrm{p}(\mathrm{dAdC})$, others & 100 & $t_{d} \propto 1 / T^{2}$, discrimination \\
Meller et al. $(2001)$ & $\mathrm{p}(\mathrm{dA})$ & $4-100$ & $I_{b}$ transition at $L \sim L_{p}, v_{\mathrm{DNA}}$ \\
Mathé et al. $(2005)$ & $\mathrm{p}(\mathrm{A})$, hairpin & $\sim 50$ & $3^{\prime} \leftrightarrow 5^{\prime}$ \\
Butler et al. $(2006)$ & $\mathrm{p}(\mathrm{A}), \mathrm{p}(\mathrm{C}), \mathrm{p}(\mathrm{A})(\mathrm{C}), \mathrm{p}(\mathrm{C})(\mathrm{A})$ & 50,75 & $3^{\prime} \leftrightarrow 5^{\prime}$, discrimination \\
\hline \hline
\end{tabular}




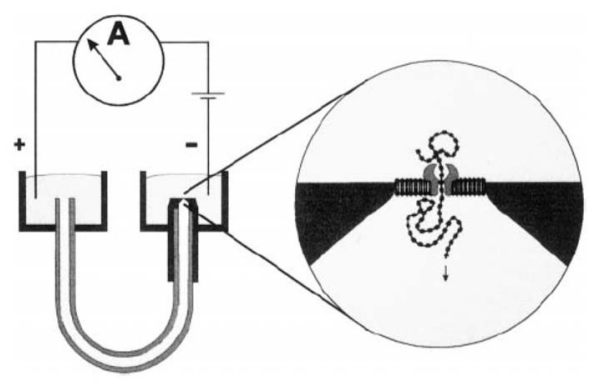

FIG. 10. A typical experimental setup for an $\alpha$-hemolysin pore. The positive voltage is applied to the trans chamber and the negative voltage to the cis one. From Akeson et al., 1999.

The experiment is then conducted by adding PNs into the cis chamber. The first experiments were done with poly(U) (Kasianowicz et al., 1996). Upon its addition, transient blockades of ionic current were observed. The blockade events actually fall into three different types distinguished by their lifetimes, as shown in Fig. 11. There are fast blockades that are independent of the poly(U) length. Thus, most likely, these are events where the strands just cover the entrance to the pore or just partially enter the pore, but do not translocate through it. However, surprisingly, both the other two types of event have lifetimes linearly dependent on the length of poly(U), and inversely dependent on the applied voltage. Kasianowicz et al. (1996) conjectured that this could be due to different translocation speeds of strands entering with either the $3^{\prime}$ or $5^{\prime}$ end. Later experiments confirmed this conjecture by examining strands composed of two homogeneous blocks and exploiting the pore's ability to distinguish between different nucleotides; see below (Mathé et al., 2005).

\section{Synthetic pores}

Synthetic pores offer additional opportunities for detection and sequencing of PNs. For instance, one can adjust the pore dimensions and properties to meet the needs of a particular experiment. Also, they open the possibility of integration of external sensors and probes, such as transverse electrodes. In addition, the parameter range for their operation is larger [although the $\alpha$-hemolysin pore is quite robust, biological pores in general are open only under certain voltages, ionic con-

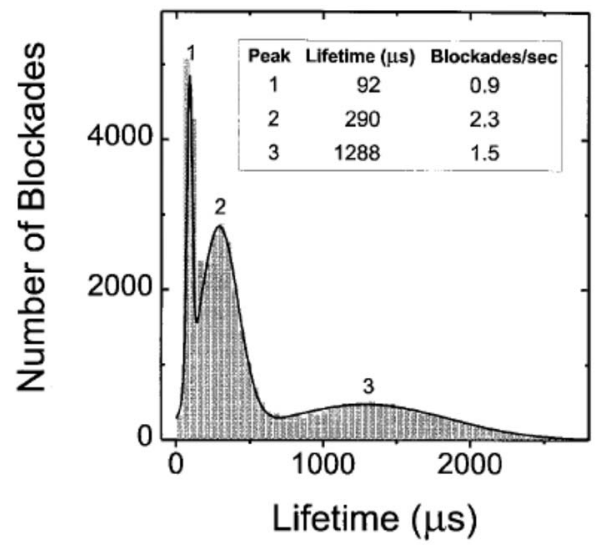

FIG. 11. Translocation duration (lifetime) of the ionic blockade events measured by Kasianowicz et al. (1996). The events fall under three different lifetimes, see the text. From Kasianowicz et al., 1996.

centrations, and temperatures (Hille, 2001)], and indeed different parameters such as salt concentration, temperature, voltage, and viscosity may be key to operating the pore in a regime where sequencing and detection are possible or most optimal, because these conditions control properties such as the translocation velocity and capture rate (see, e.g., Henrickson et al., 2000; Fologea, Uplinger, Thomas, et al., 2005). We give an overview of some of the synthetic pores made to date and the translocation experiments in Table IV.

The fabrication of synthetic nanopores is still in its nascent stages. Mainly two groups have pioneered techniques for solid-state pore fabrication. Golovchenko, working with $\mathrm{Li}$, has developed a technique using lowenergy ion beam to sculpt (ion-beam sculpting) a nanoscale hole in $\mathrm{Si}_{3} \mathrm{~N}_{4}$ (Li et al., 2001). Dekker et al. have developed a technique based on an electron beam and a visual feedback procedure (Storm et al., 2003).

The technique developed by Golovchenko et al. is shown in Fig. 12 (Li et al., 2001). One starts by creating a large-diameter pore in a solid-state membrane using a focused ion beam [Fig. 12(b)]. In their case, a $\sim 60 \mathrm{~nm}$ pore in a $\mathrm{Si}_{3} \mathrm{~N}_{4}$ membrane is first created. Then this pore is exposed to an $\mathrm{Ar}^{+}$beam, which, instead of knocking atoms off the membrane and opening the pore further, activates a diffusion process, and the pore starts to close

TABLE IV. Overview of some synthetic nanopores. Next to each pore type, their diameters and lengths are given as $\left(\left\{D_{p}\right\} ;\left\{L_{p}\right\}\right)$ in units of nanometers.

\begin{tabular}{|c|c|c|c|c|}
\hline Ref. & Pore & PN & $L(\mathrm{nt})$ & Result \\
\hline Li et al. (2003) & $\mathrm{Si}_{3} \mathrm{~N}_{4}(3,10 ; 5-10)$ & Bio-ds-DNA & 3, $10 \mathrm{knt}$ & Folded DNA $I_{b}$ events \\
\hline Chen et al. (2004) & $\mathrm{Si}_{3} \mathrm{~N}_{4}(15 ; \mathrm{n} / \mathrm{a})$ & $\lambda$-DNA, other & $48.5,10,3 \mathrm{knt}$ & $v_{\mathrm{DNA}} \propto V$, folded \\
\hline Storm, Storm, Chen, et al. (2005b) & $\mathrm{SiO}_{2}(10 ; 20)$ & Bio-ds-DNA & $\sim 6-97 \mathrm{knt}$ & $t_{d} \propto L^{1.27}$ \\
\hline Fologea, Gershow, Ledden, et al. (2005a) & $\mathrm{Si}_{3} \mathrm{~N}_{4}(4 ; 5-10)$ & Bio-ss- and ds-DNA & $3 \mathrm{knt}$ & Denaturation \\
\hline Chang et al. (2004) & $\mathrm{SiO}_{2}(4.4 ; 50-60)$ & Bio-ds-DNA & 200 & $I_{b}>I_{o}$ \\
\hline Heng et al. (2004) & $\mathrm{Si}_{3} \mathrm{~N}_{4}(1,2.4 ; 10,30)$ & $\mathrm{p}(\mathrm{dT}), \mathrm{ds}-\mathrm{DNA}$ & $50-1500$ & Length discrimination \\
\hline
\end{tabular}



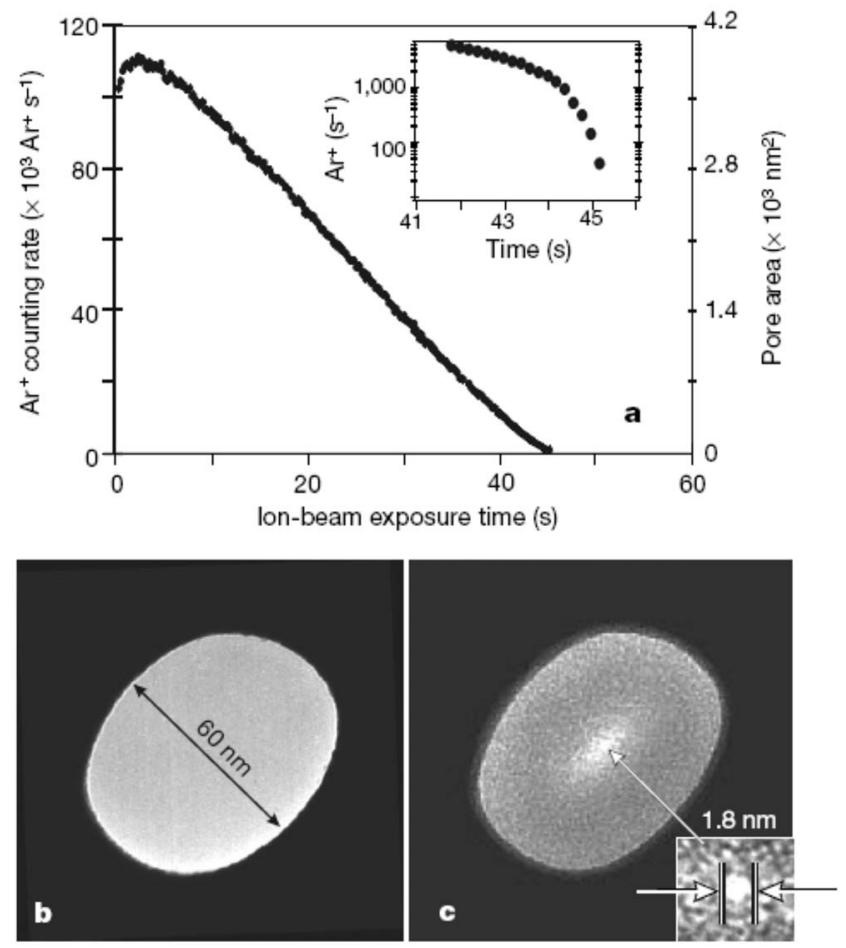

FIG. 12. Fabrication technique of Li et al. (2001) for creating nanoscale solid-state pores: a $\mathrm{Si}_{3} \mathrm{~N}_{4}$ membrane with a large pore is first created (b). An ion beam is then focused at this large pore, activating a diffusion process that closes the hole (c). The current of $\mathrm{Ar}^{+}$decreases as the pore shrinks (a), the monitoring of which can be used to control pore size. From Li et al., 2001.

(Li et al., 2001). The $\mathrm{Ar}^{+}$current coming through the pore is directly dependent on the pore area. Thus, one can measure the $\mathrm{Ar}^{+}$current, shown in Fig. 12(a), as a function of time, and use this to controllably shrink the pore down to the nanometer scale [Fig. 12(c)]. The precise composition of silicon and nitrogen around the pore is not known (Li et al., 2001).

Dekker and co-workers have developed a similar technique based on the idea of pore diameter reduction upon irradiation (Storm et al., 2003). They used a highenergy electron beam and $\mathrm{SiO}_{2}$ membrane. The initial, larger pore can be formed in different ways. But once the initial pore is made, a transmission electron microscope (TEM) is used to shrink it. By using the imaging capability of the TEM, one can visually watch the pore diameter decrease (Storm et al., 2003). Since the rate of size reduction is slow $(0.3 \mathrm{~nm}$ per minute in these experiments), the visual images can be used to monitor the diameter of the pore and the process stopped when the desired size is reached. The resolution of the microscope $(\sim 0.2 \mathrm{~nm})$ then sets the limit of accuracy for reaching the desired size. However, due to the roughness of the surface, one can control the pore size only to about $1 \mathrm{~nm}$ dimensions (Storm et al., 2003).

Synthetic pores have properties that differ from those of the biological pores. The surfaces can be charged in solution, creating an additional complication for under- standing ionic and PN transport. For instance, siliconoxide surfaces can have a negative surface charge density on the order of $10^{-2} e / \AA^{2}$ in aqueous solution (Chang et al., 2004). This would give quite a substantial charge within any reasonably sized nanopore, and would have to be neutralized by counterions. Biological pores, though, can have unusual potential profiles that may have internal sites with trapped charges (Hille, 2001).

The observation that high-energy beams can cause pores to shrink is an interesting phenomenon in and of itself, in addition to its implications for the fabrication of nanoscale structures and pores. We mention here that Storm et al. (2003) observed that there is a transition from shrinking to growing as the pore diameter is increased, and they explained this observation by a surface tension effect.

\section{DNA translocation}

One can divide the translocation of PN through a pore into two categories: universal properties of polymer dynamics (entropic forces, Brownian motion, charges, and screening) and specific properties that rely on the atomic compositions of the nucleotides (e.g., interaction potentials with the pore surface). Depending on the quantity under consideration, and the parameter regimes of the device, either one or both of these categories will be important.

\section{Universal properties}

The two basic properties of charged polymer dynamics are the processes of capture and translocation. The capture of the polymer will depend on the diffusion of the polymer from the bulk to the pore and on local effects around the pore, such as the electric field and interactions between the entrance of the pore and the polymer. The capture rate will depend on concentration and applied bias (Henrickson et al., 2000; Nakane et al., 2003), as well as what molecule is under investigation, and will have repercussions on the ability to detect and sequence. The translocation through the pore will be driven by the applied bias, but depends on many factors, including the polymer-pore interactions, ionic effects, and viscous drag. However, two properties are common to translocation in nanopores, namely, the effective charge and screening of polymers within the pore and the related issue of where the applied voltage drops.

In the absence of a polymer within a pore, one expects that the majority of the voltage drop between the two ion chambers occurs in the pore since it has a resistance higher than the surrounding solution. However, depending on its shape (which depends on whether the pore is biological or synthetic), one would expect that the presence of a polymer in the pore can significantly change the voltage drop of the system. For instance, with the $\alpha$-hemolysin pore in Fig. 9, one might expect that, when the polymer creates a much higher resistance, this occurs primarily in the region of the pore that is roughly $2 \mathrm{~nm}$ in diameter. Thus, the potential profile will change and 


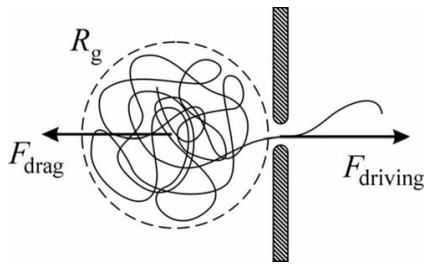

FIG. 13. Drag of a coiled-up polymer outside a pore. From Storm, Chen, Zandbergen, et al., 2005.

drop more significantly over the stem. This would cause the applied bias to act most significantly on the nucleotides (or charged polymer units) that are located within the stem.

There is some indirect evidence that this is the case. Meller et al. (2001) showed that there is a transition in velocity dependence of the translocation process. Above about 12 nucleotides, the velocity of the strands is independent of their length, suggesting that the bias is pulling only on a finite number of charges and is acting solely against the drag of the polymer within the stem. Below about 12 nucleotides, the velocity increases (in a highly nonlinear way) with decreasing length. For 12 nucleotides, the contour length is approximately $5 \mathrm{~nm}$, and thus is in agreement with the pore stem length. This transition is explained by a model of polymer translocation through a pore of finite length (Slonkina and Kolomeisky, 2003), which gives a transition in velocity when the polymer's length is equal to the pore length.

Another interesting fact is that there is a transition in polymer dynamics based on the pore size and porepolymer interaction. For pores with diameter roughly the size of the polymer width, one expects strong interactions of the polymer with the walls of the pore, thus strongly increasing its resistance to flow. When this is the case, the time scale of polymer translocation $t_{d}$ will be controlled by this resistance, which will dominate over other factors, such as polymer unraveling and drag outside the pore (see Fig. 13). In this regime, one expects the translocation duration to be linearly proportional to polymer length (for polymer lengths much larger than the pore length). Indeed, this is what has been measured (Kasianowicz et al., 1996). On the other hand, if the pore is very wide (or there is little polymer-pore interaction), the translocation time can be controlled by other factors, and one does not expect this time to have a linear relation with the polymer length.

Such a nonlinearity has been observed and explained by Storm, Chen, Zandbergen, et al. (2005) and Storm, Storm, Chen, et al. (2005). These authors observed that the (most probable) translocation duration scales as

$$
t_{d} \propto L^{1.27}
$$

for unfolded ds-DNA translocation. By considering a model that accounts for the driving electric force and the hydrodynamic drag force on the coiled-up polymer outside the pore, Storm, Storm, Chen, et al. (2005) obtained a translocation duration that scales with polymer length as $L^{1.22}$, which compares well with the experimental value. The unusual exponents come from the fact that the radius (of gyration) of a polymer coil scales as a function of its length with noninteger exponent, and depends on the polymer type (self-avoiding, etc.). The drag force will be dependent on the surface area of the coiled polymer. Most sequencing technologies will probably have to deal with small pore diameters, and thus in the regime where the drag of the polynucleotides within the pore is significant. Therefore, in these devices the translocation duration should scale with the length of the polynucleotide. More generally though, this result shows that, if one wants to use a nanopore as a polymer detector, the latter has to be first calibrated to take into account specific details that affect the measured characteristic signals [similar calibration has to be performed in the case of sequencing (Lagerqvist et al., 2006); see Sec. V.A.2].

The linearly extended polymer may not be the only one able to translocate. If the pore diameter $D_{p}$ is large enough to accommodate multiple strands, there could be ionic blockade events corresponding to folded polymers. In this case, we analyze the forces on the DNA nucleotide pairs, and, for folded DNA, on the nucleotides that fall within the pore. This analysis highlights the complexity of polymer dynamics in nanopores. If the applied voltage drops solely over the pore, the driving force on the polymer in a given region of the pore will be

$$
F_{\text {driving }}(m) \approx m F_{\text {driving }}^{o},
$$

where $m$ is the number of folds in the region and $F_{\text {driving }}^{o}$ is the driving force on a single nucleotide pair,

$$
F_{\text {driving }}^{o}=z_{\text {eff }} E,
$$

where $z_{\text {eff }} \approx-0.5 e$ (see Sec. V.C) is the effective charge on the nucleotide pair and $E$ is the electric field. If porepolymer interactions are responsible for the majority of the drag force, then one also obtains a similar relation

$$
F_{\text {drag }}(m) \approx m F_{\text {drag }}^{o},
$$

where, e.g., $F_{\text {drag }}^{o} \approx \eta v$, with $\eta$ a coefficient that is proportional to the surface area for contact of the polymer and pore surface (that is the reason why $\eta \rightarrow m \eta$ for the folded polymer) and $v$ is the polymer velocity. For a constant polymer velocity, ${ }^{4}$

\footnotetext{
${ }^{4}$ This, of course, assumes that there is such a thing as a "constant velocity" for this nanoscale object. This is not strictly true, and estimates of drag and subsequent analysis may be affected by fluctuations.
} 

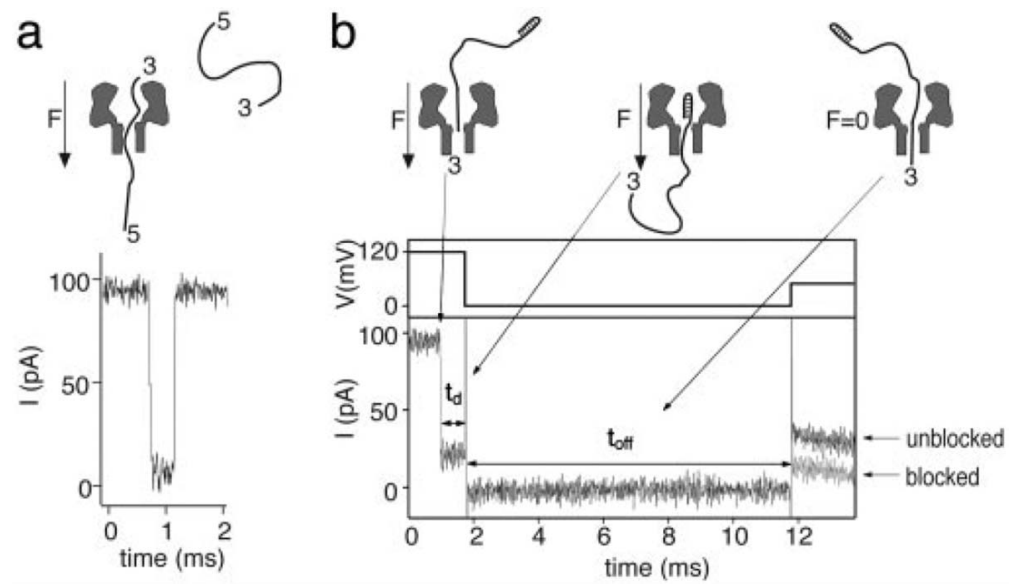

FIG. 14. Schematic of a hairpin experiment with an $\alpha$-hemolysin pore. (a) A typical experiment on ss-DNA can pull the strand through from $3^{\prime} \rightarrow 5^{\prime}$ or from $5^{\prime} \rightarrow 3^{\prime}$, leading to blockades with different characteristics. (b) The double-stranded portion of the DNA hairpin cannot transverse the pore. Thus, the hairpin can be pulled as far as possible into the pore, and one can turn off the pulling voltage for some time $t_{\text {off }}$ after which one can check whether the strand is still in the pore by applying some small probing voltage. From Mathé et al., 2005.

$$
F_{\text {drag }}=F_{\text {driving }} \text {, }
$$

and thus the factors of $m$ will cancel, and $v$ would be the same regardless of whether the polymer is folded or unfolded $\left(v=v_{o}\right)$. Now consider a polymer that has a region of length $L_{m}$ of $m$ folds, with $L_{m} \gg L_{p}$. If we suppose volume exclusion to be responsible for ionic blockade (see the next section), the blockade current of the folded and unfolded polymer will be related by

$$
I_{o}-I_{b}^{m}=m\left(I_{o}-I_{b}^{o}\right),
$$

where $I_{o}$ is the open pore current, $I_{b}^{o}$ is the blockade current for an unfolded polymer, and $I_{b}^{m}$ is the corresponding quantity for the polymer with $m$ folds. Given Eq. (6), one finds that the total charge blocked for the translocation of the folded polymer region is equal to the total charge blocked by a linearly extended region of length $m L_{m}$, which contains the same number of nucleotides,

$$
\left(I_{o}-I_{b}^{m}\right) \frac{L_{m}}{v}=\left(I_{o}-I_{b}^{o}\right) \frac{m L_{m}}{v_{o}} .
$$

This is what has been dubbed the rule of constant event charge deficit (ECD) (Fologea, Gershow, Ledden, et al., 2005),

$$
(\mathrm{ECD}) \equiv \Delta I_{b} t_{d}=\mathrm{const},
$$

where $\Delta I_{b}=I_{o}-I_{b}$ and $t_{d}$ is the translocation time. In experiments where this holds, it would seem to justify an excluded-volume model for the blockade current.

This phenomenon has been observed in experiments with a 10-nm-diam $\mathrm{Si}_{3} \mathrm{~N}_{4}$ pore (5-10 nm thick) and $10 \mathrm{kbp}$ ds-DNA (Li et al., 2003). A smaller $\mathrm{Si}_{3} \mathrm{~N}_{4}$ pore of $4 \mathrm{~nm}$ diameter also showed a set of events that were distributed according to this rule (Fologea, Gershow, Ledden, et al., 2005). However, these observations were only for larger pores (4 and $10 \mathrm{~nm}$ for ds-DNA, $4 \mathrm{~nm}$ for ss-DNA), and thus they are not necessarily indicative of the physics of smaller pores. There are also events that do not fall on curves of constant ECD (Fologea, Gershow, Ledden, et al., 2005). These seem to be events where the ds-DNA molecules temporarily bond to the walls of the pore or have some other factor controlling their velocity (besides viscous forces), such as untangling of the ds-DNA outside of the pore.

To get the constant event charge deficit, one has to assume that a pore-polymer interaction or, at least, a polymer interaction specific to the pore region (e.g., an electrostatic drag induced by the confinement) is causing the drag, which will give the relation (4). With this type of drag, one expects the translocation duration to scale as $L$, the length of the polymer. However, Storm, Chen, Zandbergen, et al. (2005) found that the most probable translocation duration scales as in Eq. (1). This seems to indicate that it is not the polymer-pore interaction creating the drag but rather the externally jumbled polymer. There are many possible physical explanations for such a discrepancy, and other experiments are needed to have a complete understanding of these effects.

\section{Specifics}

Here we give just one example of an interesting specific property observed for PN experiments with $\alpha$-hemolysin pores. We previously discussed the results of Kasianowicz et al. (1996) (see also Akeson et al., 1999), which raised an intriguing question about the directionality of translocation and capture of the PN. Are these two processes dependent on which end, the $5^{\prime}$ or 3', translocates first? Recently, both experiments and MD simulations have confirmed directionalitydependent translocation. Mathé et al. (2005) performed experiments on DNA hairpins, as shown in Fig. 14 (see also Henrickson et al., 2000). The experiment was performed by pulling the hairpin into the pore, measuring the ionic blockade, then turning off the pulling voltage for a time $t_{\text {off }}$ and using a small probing voltage to detect whether the pore was still blocked. These authors found that hairpins with the $3^{\prime}$ end dangling (translocating $3^{\prime}$ $\rightarrow 5^{\prime}$ ) have a lower blockade current than the $5^{\prime}$ end (translocating $5^{\prime} \rightarrow 3^{\prime}$ ); see Fig. 14. Further, these two blockade currents are in good agreement with the two peaks found from performing the typical single-stranded experiment (with no hairpin). This indicates that early experiments obtaining translocation events with two dif- 
ferent blockade currents were measuring the difference in orientation of the strands. In addition, the diffusion and velocity (when pulled) of the hairpin out of the pore were found to be much slower for the $3^{\prime}$ end entering, e.g., when the strand is translocating $5^{\prime} \rightarrow 3^{\prime}$. This is also a good indicator that the early observation of two lifetimes (with different capture rates) of translocation events was likely due to different orientation of the translocating DNA.

In addition, Mathé et al. (2005) performed MD simulations that demonstrated, in a pore, the bases on the DNA tilt toward the $5^{\prime}$ end. This explains the slower motion of the DNA, because when moving the $5^{\prime}$ end first there is additional "mechanical" friction. This phenomenon will be important not only for detection of different types of polynucleotides, but also for any of the sequencing proposals described below. For instance, if there is a different average base orientation depending on which end enters first, the current distributions of the bases (see Sec. V.A.2) may also be different depending on whether transverse control erases the directional differences. One may also ask whether this difference in $5^{\prime}$ and $3^{\prime}$ orientations will be present in a synthetic pore, or whether it depends on properties specific to the $\alpha$-hemolysin pore. Further, the interplay between this effect and that of secondary structure is still unclear, pointing to the need for more experimental and theoretical investigation.

\section{Pore and ion electronics}

From the above discussion, we conclude that ionic currents through the nanopore are important for the detection of polynucleotides. However, much is still not understood about the translocation of ions themselves. The interplay between volume exclusion, steric blockage, electrostatics, solution-ion dynamics, and interaction with the pore itself is quite complex and poorly understood.

\section{Ionic and blockade currents}

In part, the blockade current forms due to "volume exclusion." The polynucleotide occupies part of the pore's volume, which partially blocks ions from occupying the pore. This reduces the number of charge carriers and thus the current. How much of the volume is occupied by the PN depends on the structure and composition of the strand. PNs with helical secondary structure could block more of the pore area if the secondary structure stays intact. There are additional questions on how much other factors, such as, e.g., DNA-pore interactions and electrostatics, contribute to the blockade.

To get an idea of the contribution of volume exclusion to the blockade current, we can use a steady-state form of the Nernst-Planck equation (Barcilon et al., 1992; Chen et al., 1992; Schuss et al., 2001; Coalson and Kurnikova, 2005)

$$
J_{z}=e \mu n E_{z}-e D \frac{\partial n}{\partial z}
$$

for the charge flux $J_{z}$ in the $z$ direction, where $e$ is the electric charge (assuming monovalent cations), $\mu$ is the ion mobility, $n$ is the charge-carrier density, $D$ is the diffusion coefficient, and $E_{z}$ is the driving field in the $z$ direction. The general assumption that goes into the NP equation is that of a continuous ionic distribution contained within a continuum dielectric. The particular form of Eq. (9) assumes that there is no free-energy barrier to ion transport through the pore, and thus it is a great start to examine whether volumetric effects are responsible for the blockade current.

In the absence of any substantial diffusive term, the open pore current reduces to

$$
I_{o}=e \mu n E_{z},
$$

where $E_{z} \approx V / L_{p}$ (the voltage $V$ drops mostly over the pore length $L_{p}$ ). The quantity $e \mu n=\sigma$ is the conductivity of the pore.

If volume exclusion were the sole factor in the change in current, one could write

$$
n_{b}=\frac{V_{p}-V_{N}}{V_{p}} n=F n,
$$

where $n_{b}$ stands for the carrier density during a blockade event, $V_{p}$ is the pore volume for one repeat unit of the $\mathrm{PN}$, and $V_{N}$ is the volume of a nucleotide (see Table I). For the fraction reduction in current from open $\left(I_{o}\right)$ to blocked $\left(I_{b}\right)$ pore, we then get

$$
\frac{I_{o}-I_{b}}{I_{o}}=1-F .
$$

More simply, if one assumes that the carrier density is the same, but that part of the pore area $A_{o}$ has been blocked, then $\left(I_{o}-I_{b}\right) / I_{o}=A_{b} / A_{o}$, with $A_{b}$ the portion of the area that is blocked. If we assume the same length $l$ for both the unblocked and blocked pores, this relation will also give Eq. (12). The whole analysis requires a regularity in the orientation of the blocking species, which may not be completely correct.

For random coil (extended) ss-DNA, $F$ can be matched with the volume fraction from Table I. The volume of the nucleotides in Table I is for DNA nucleotides, but should be essentially the same for RNA nucleotides, and the repeat units in the PN, whether charged or passivated. This is because (i) the extra hydroxyl group has a marginal effect on molecular volume, and (ii) the secondary structure should also not change the volume occupied per nucleotide. The reason for the latter is simple: the molecular volumes were computed with the van der Waals radii, which are of the same order as half the base separation in stacked secondary structures. For example, the van der Waals radius is $1.7 \AA$, but the base separation is $\sim 3 \AA$. Thus, one may expect that secondary structure can slightly increase or decrease the nucleotide volume, but only by a small fraction. 
Consider now random coil poly $(\mathrm{C})$, or poly $(\mathrm{dC})$, within the $\alpha$-hemolysin pore. We take $15 \AA$ as an estimate for the pore radius, and suppose the $\mathrm{PN}$ is maximally linearly extended such that each nucleotide occupies a $7 \AA$ length of pore. ${ }^{5}$ In this case, $1-F=0.26$ for cytosine, which does not come close to the blockade values [>90\% (Akeson et al., 1999)]. When the secondary structure changes, this will change the length of the pore that each nucleotide occupies. If, for instance, the bases stack at a distance $3.4 \AA$ [poly $(\mathrm{C})$ stacks at $3.1 \AA$ (Arnott et al., 1976)], giving a minimum occupation length in the pore, then $1-F=0.52$. This is still much smaller than the blockade values found experimentally.

Of course, one may need to compute molecular volumes including the hydration of the nucleotides (Deamer and Branton, 2002) and also divide the pore into "good" and "bad" volumes, as there may be bound water molecules on the walls. This would certainly give a larger fractional reduction in volume. However, defining volumes with the hydration layers included may not be accurate in the dynamical pore environment, as translocating ions may temporarily share hydration layers with the nucleotides. But this raises some interesting questions about the interaction of the different bases with the nearby water and ions. For instance, do the bases interact sufficiently different with the water that the free volume, including hydration layers, distinguishes the bases better than in Table I, or is it the reverse? One would expect the latter, because the hydration layers probably smooth over differences in the bases, and the backbone is likely the main location for bound water molecules.

Regardless, the argument seems strong enough that it points to other effects (interactions with the pore walls and electrostatics) as the main cause of the blockade current for DNA. Other polymers, especially neutral ones such as polyethylene glycol, may show different behavior. However, the blockade is still a complex phenomenon. For example, poly $(\mathrm{C})$ and poly $(\mathrm{dC})$ have the same base, but the interaction with the pore walls and dynamics could change due to the secondary structure. In this case, the more structured poly $(\mathrm{C})$ may create dynamical charge traps within the pore (a steric effect), thereby reducing the current further than for poly $(\mathrm{dC})$. Also, poly $(\mathrm{dC})$ creates larger current blockages than poly(A) $[89 \%$ and $86 \%$ for poly $(\mathrm{dC})$ compared to $85 \%$ and $55 \%$ for poly(A) (Akeson et al., 1999)]. Since C has a much larger association with the lysine in the $\alpha$-hemolysin pore (Bruskov, 1978), interactions may be the determining factor.

Further, there are other effects to consider. Volume exclusion creates an additional electrostatic barrier due to the increased confinement of the ions. Thus a small change in volume can decrease the current because the associated free-energy change "deactivates" many ions for transport (see below). Additionally, PNs remain

\footnotetext{
${ }^{5}$ This would give about seven nucleotides in the pore stem, but there may be more, depending on secondary structure.
}

charged within the pore (Keyser et al., 2006; Cui, 2007). How does this affect the blockage of coions and the flow of counterions? ${ }^{6}$ We are not aware of a complete answer to this question.

\section{Electrostatics}

Another issue related to the ionic currents is how the electrostatic environment of the pore affects transport. Some partial answers can be found by looking at simplified models of the pore environment, and using them as input to the full NP equation. ${ }^{7}$ For instance, one could take an appropriate free-energy potential, and possibly position-dependent dielectric constants and mobilities. In the case in which the pore diameter is small, the freeenergy change can be quite large and thus significantly suppress ion transport.

In the regime of large free-energy change, we consider a scenario in which the two chambers have an equilibrium concentration of ions at their respective shifted chemical potentials. Most of the ions do not have the energy to move across the pore. So we consider an activated process in which the number of ions available for transport is given by

$$
n=n_{o} e^{-\Delta F_{b} / k T},
$$

where $n_{o}$ is the bulk density and $\Delta F_{b}$ represents the freeenergy barrier due to both an electrostatic energy barrier and an entropic barrier. For large pores, both of these contributions will be negligible as the electric field from the ion is still embedded in a large volume of water and there is still a large phase space for ions to occupy.

For smaller pores, one can think of the simplified model shown in Fig. 15. The general concept is to look at the pore as a continuum large dielectric (water) region of space surrounded by a material of low dielectric $\left(\mathrm{SiO}_{2}, \mathrm{Si}_{3} \mathrm{~N}_{4}\right)$, thus creating an electrostatically quasione-dimensional structure (Teber, 2005; Zhang et al., 2005). Under this simplified picture, the electrostatic and entropic components to $\Delta F_{b}$ can be computed. Zhang et al. (2005) found that this simplified model gives a tremendously suppressed ionic current due to the large free-energy change of ions entering the pore. However, they also found that any significant presence of wall charges, which are present in experimental systems such as silicon dioxide, would decrease the free-energy barrier and thus increase the ionic conductance (Zhang et al., 2005). Recently many researchers have looked at nanopore-ion-DNA electrostatics from this point of

\footnotetext{
${ }^{6}$ An unusual phenomenon of current enhancement due to DNA translocation was found in some experiments (Chang et al., 2004, 2006; Fan et al., 2005). This has been explained in terms of an enhanced counterion current due to the presence of the backbone charge of the DNA, which at low ionic concentrations overpowers the volume exclusion.

${ }^{7}$ One can also question the validity of that equation because inhomogeneities at the nanoscale may be important. We are not aware of a critical study of this point.
} 


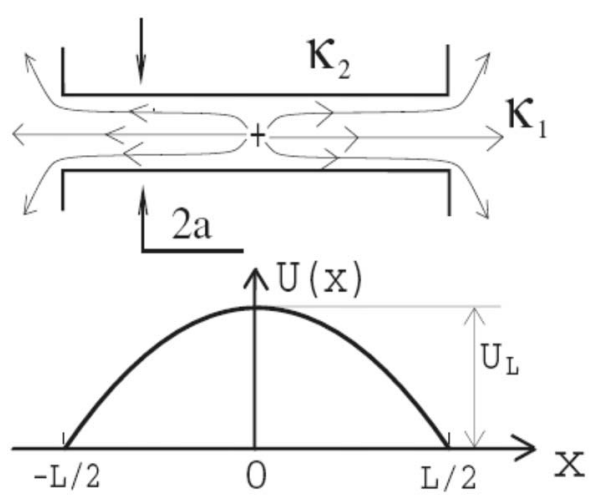

FIG. 15. Continuum pore model used by Zhang et al. (2005). The dielectric constants are such that $\kappa_{1} \gg \kappa_{2}$ so that all field lines of a charge in the pore run down the pore axis. The lower panel shows the electrostatic energy barrier for a single charge to transport from the bulk into the pore. From Zhang et al., 2005 .

view (Zhang et al., 2005; Bonthuis et al., 2006; Kamenev et al., 2006; Zhang, Kamenev, and Shklovskii, 2006; Zhang and Shklovskii, 2007).

Lagerqvist et al. (2007c) looked at the microscopic effects of the electric fields and solution structure. If one examines the field of a single anion surrounded by a spherical droplet of water, this looks very different from what one would expect from a bulk dielectric. ${ }^{8}$ The field obtained from MD simulations, and averaged over time and solid angle, is shown in Fig. 16. One can clearly see the formation of a nanoscale structure around the ion: the water molecules start to form layers (called hydration layers). For instance, for the $\mathrm{Cl}^{-}$we show here, there are on average six water molecules in the first layer that orient their dipoles very strongly toward the anion. These hydration layers are only partially destroyed within a pore (Lagerqvist et al., 2007c), and the free-energy barrier has an electrostatic contribution given by the partial destruction of the hydration layers. As the pore radius is increased, there is a critical radius that allows a full hydration layer to be transported through the channel. This creates a strong nonlinear dependence of the free-energy barrier as a function of pore radius and also ionic concentration. Effects like the above illustrate the need to take a microscopic approach to understanding nanopore electronics, and foreshadows novel physical phenomena that will be observed at the interface between solids and liquids (and biomolecules) in the nanometer regime.

\section{SEQUENCING AND DETECTION}

We have reviewed the physical characteristics of the bases and polynucleotides, and also discussed nanopores

\footnotetext{
${ }^{8}$ This is, of course, due in part to the fact that macroscopic electrostatics requires averaging the electric field over large regions of space of at least $10 \mathrm{~nm}$ (Jackson, 1998). It is also due to the formation of hydration layers as a consequence of the strong local electric field.
}

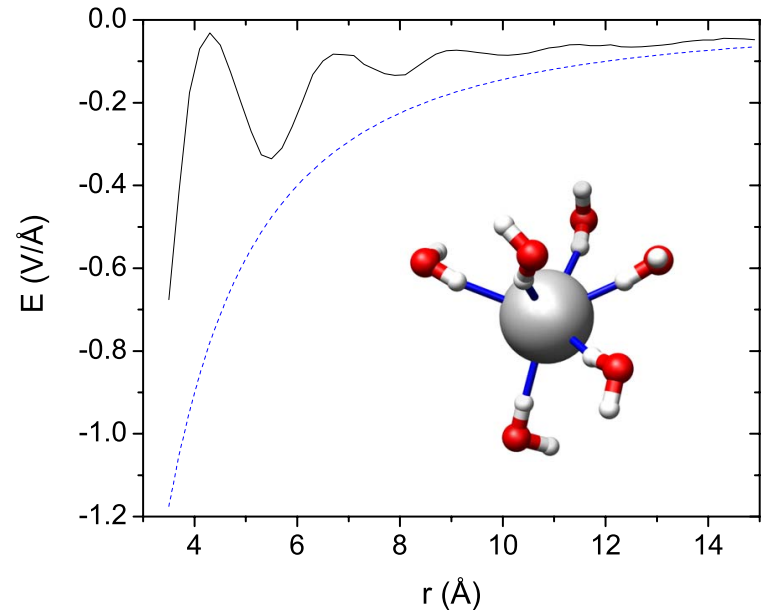

FIG. 16. (Color online) Microscopic electric field of a single anion in a spherical water droplet of radius $25 \AA$ (solid line). The dashed line is the bare field. The inset shows a model of the first hydration layer.

and DNA translocation. Thus, we are now in a position to examine in more detail the physical mechanisms by which DNA can be detected and sequenced. Very loosely, we divide the physical mechanisms into three categories: electronic, optical, and force methods. In this section, we discuss several of the proposed methods in terms of the physical differences of the bases, and outline any experimental and theoretical results pertinent to either measuring these property differences or constructing a working apparatus.

\section{A. Electronic detection}

The electronic methods proposed so far are based on the ionic blockade current in the nanopore (Kasianowicz et al., 1996; Deamer and Branton, 2002), embedding nanoscale electrodes in the nanopore to measure transverse transport across ss-DNA (Zwolak and Di Ventra, 2005; Lagerqvist et al., 2006; Lee and Meller, 2007), or measuring the voltage fluctuations in a capacitor across the longitudinal direction of the pore (Heng, Aksimentiev, Ho, Dimitrov, et al., 2005; Gracheva, Xiong, Aksimentiev, et al., 2006). Three specific proposals using these techniques are shown in Fig. 17.

\section{Ionic blockade}

With the promising first results of Kasianowicz et al. (1996), it was speculated that it may be possible to sequence DNA by measuring the ionic blockade in a very particular pore under the right conditions (see Kasianowicz et al., 1996; Deamer and Akeson, 2000; and Deamer and Branton, 2002). With this idea in mind, researchers started to examine what kind of information can be extracted from the nanopore experiments. Indeed, other experiments with $\alpha$-hemolysin pores have shown that the ionic blockade can be used to detect 


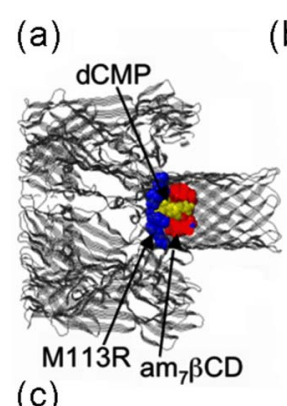

(b)

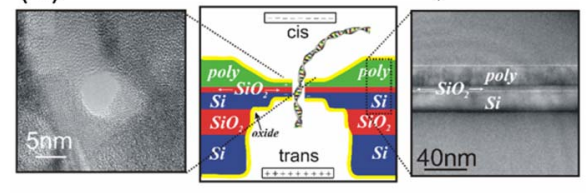

FIG. 17. (Color online) Example nanopores for electronic detection of DNA. (a) Engineered $\alpha$-hemolysin pore used to detect nucleotides via their ionic blockade. Adapted from Astier et al., 2006. (b) Schematic of two electrodes embedded in a nanopore. As a PN translocates through the pore, electrodes drive a current across the nucleotides in the transverse direction. The current across each of the different nucleotides provides an electronic signature of the base. $E_{\|}$is due to the voltage pulling the DNA through the pore. $E_{\perp}$ is the field perpendicular to the electrode surface and is provided by the applied voltage across the electrodes and any additional external capacitor. From Lagerqvist et al., 2007b. (c) Middle panel: schematic of a nanopore through a capacitor made of doped polycrystalline silicon- $\mathrm{SiO}_{2}$-doped crystalline silicon. The $\mathrm{SiO}_{2}$ can be as thin as $0.7 \mathrm{~nm}$ (Gracheva, Xiong, Aksimentiev, et al., 2006), which is about the spacing of nucleotides in extended ss-DNA. From Gracheva, Xiong, Aksimentiev, et al., 2006.

blocks of nucleotides, and that the blockade characteristics give information on secondary structure and directionality $\left(5^{\prime}, 3^{\prime}\right)$ of $\mathrm{PN}$ translocation.

For instance, by looking at RNA homopolymers poly(A), poly(U), and poly(C), Akeson et al. (1999) demonstrated that the blockade current and the translocation duration can be used to distinguish between the types of base present in the homopolymers. At $120 \mathrm{mV}$ bias, it was found that $\operatorname{poly}(\mathrm{U})$ translocation takes 1.4 and $6 \mu \mathrm{s} / \mathrm{nt}$ for two translocation event types, compared to $22 \mu \mathrm{s} / \mathrm{nt}$ for poly(A). Yet, they both can give blockade currents of essentially identical magnitude (approximately $85 \%$ blocked). On the other hand, poly(C) was found to give $95 \%$ and $91 \%$ blockade of the ionic current, and to translocate at $\sim 5 \mu \mathrm{s} / \mathrm{ns}$.

An interesting feature of the above results is that poly $(\mathrm{C})$ gives a larger blockade of the ionic current than poly(A) even though it is a smaller base (see Table I). Akeson et al. (1999) suggested that this could be due to the secondary structure of poly $(\mathrm{C})$, which at neutral $p \mathrm{H}$ and room temperature has a helical structure that is $1.3 \mathrm{~nm}$ in diameter (Saenger, 1988). This size is small enough to fit into the $\alpha$-hemolysin pore without unraveling. To test this, these authors compared $\operatorname{poly}(\mathrm{C})$ to poly $(\mathrm{dC})$, its DNA counterpart. Poly $(\mathrm{dC})$ secondary structure is not as stable as poly $(\mathrm{C})$, so differing results for $\operatorname{poly}(\mathrm{dC})$ and $\operatorname{poly}(\mathrm{C})$ would give evidence for secondary structure as the cause. Akeson et al. (1999) indeed found that poly $(\mathrm{C})$ blocked more ionic current than poly $(\mathrm{dC})$. Thus the secondary structure of poly $(\mathrm{C})$ is the likely cause of the larger blockade. This, however, leaves an open question raised earlier in Sec. IV.D: Why does the secondary structure cause a larger blockage of current?

The slow translocation of poly(A) is also thought to be due to secondary structure. Poly(A) has a secondary helical structure, which, unlike poly $(\mathrm{C})$, makes it too big to translocate through the pore (Akeson et al., 1999). Thus, poly(A) takes extra time to unravel and go through the pore. On the contrary, poly(U) does not have any secondary structure under the reported experimental conditions (see discussion in Kasianowicz et al., 1996 and Akeson et al., 1999).

It has also been shown that a PN with two homogeneous blocks $\mathrm{A}_{30} \mathrm{C}_{70}$ gives ionic blockade events with a stair structure: a higher current is present when the homogeneous block of A's is present in the pore, but this current decreases when the C's are in the pore (Akeson et al., 1999). Although far from single-nucleotide resolution, this shows that one can obtain some internal information about the strand from the nanopore. This result, together with using the average blockade current, average translocation time, and the temporal dispersion (Meller et al., 2000) to distinguish individual molecules, shows some of the promise of nanopore technology in polynucleotide detection.

Later, Fologea, Gershow, Ledden, et al. (2005) took advantage of the ability of solid-state pores to work under many conditions by examining DNA translocation at many different $p \mathrm{H}$ 's in order to detect the ds-DNA denaturation into ss-DNA. As they increase the $p \mathrm{H}$ from 7 to 13 , these authors found that there is a rather abrupt transition when the current blockade drops by roughly a factor of 2 (Fologea, Gershow, Ledden, et al., 2005). That this is due to denaturation is confirmed by measurement of the optical absorbance, which shows that denaturation occurs at around $p \mathrm{H}=11.6$. These are examples of physical processes that can be studied with, and detection capabilities that can be achieved by, nanopore technology.

In order to actually sequence a strand of DNA, singlebase resolution is required. In this case, the different sizes of the bases, and the different interactions between the bases and pore, have to be detected. For direct use of the ionic current as an indicator of the base present at a location in a polynucleotide (i.e., for sequencing), the nanopore must have a length $L_{p}$ of roughly one nucleotide $(<1 \mathrm{~nm})$ and diameter somewhere between 1 and $2 \mathrm{~nm}$.

If the ionic blockade is solely due to excluded volume, then one would expect that the differences of the bases in a 1.5-nm-diam, 0.7- nm-long pore would be just a few percent, as shown in Table I. The noise of the current itself, both intrinsically (due to ionic fluctuations) and due to structural fluctuations of the nucleotides, is likely 
to be much larger than this. ${ }^{9}$ For instance, the fluctuation of the ionic blockade current for homogeneous sequences is about $30 \%$ of the average current (see the figures in Meller et al., 2000). The noise for a single base should be larger than for the sequences. Intuitively, comparing to an $\alpha$-hemolysin pore with about 10 bases in the stem, one expects the noise for one base to be larger by a factor of about $\sqrt{10}$. This would mean noise on the order of a $100 \%$ of the average current. There may be possibilities, such as changing conditions like temperature, $p \mathrm{H}$, etc., to minimize secondary structure effects and make the circumstances more amenable to sequencing. But even in the best case scenario, where only the pore-located nucleotide is controlling the ionic blockade current, it may not be possible to successfully distinguish the bases; (see also the arguments presented in Sec. IV.D).

Nonetheless, more recent experiments have shown that single-base differences can be detected in strands. For instance, Vercoutere et al. (2001) looked at several different length DNA hairpins that contain no singlestranded portion. The hairpins initially block the vestibule part of the $\alpha$-hemolysin pore, and can be pulled through the pore only when the double strand temporarily unravels. These authors found that one can discern two hairpins with only a difference of one base in the loop and also two hairpins with only a mismatch as a difference. Both the vestibule blockade current and the vestibule blockade duration were found to be different. The hairpin with the base pair mismatch is much more likely to unravel, and therefore spends much less time in the vestibule before translocation (a factor of 100 less according to these experiments). A similar situation exists for the shorter loop hairpin, which is under much more strain.

Using ionic blockade, the same group has looked further at hairpin differences, computer-learning algorithms for distinguishing signatures, and the physics of hairpins within pores (Vercoutere et al., 2001, 2003; Winters-Hilt et al., 2003; DeGuzman et al., 2006). Another group obtained similar findings for a different type of hairpin experiment, where a single base difference in a single-strand leg of a hairpin could be detected (Ashkenasy et al., 2005). It is important to note, however, that these experiments do not actually achieve single-base resolution as required for sequencing.

Without amplification of the bases, it is unlikely that a strand of bases can be sequenced with the bare ionic current. However, there is potential to go beyond the normal current blockade experiments by creating designer pores (Bayley and Cremer, 2001; Siwy et al., 2005). For instance, introducing a foreign molecule into the pore or by using a pore that has some chemically specific

\footnotetext{
${ }^{9}$ Molecular-dynamics simulations of Aksimentiev et al. (2004) indicated that fluctuations in the ionic current due to structural changes are larger than the differences between bases. These structural changes are in part caused by interaction with the pore surface.
}

(a)
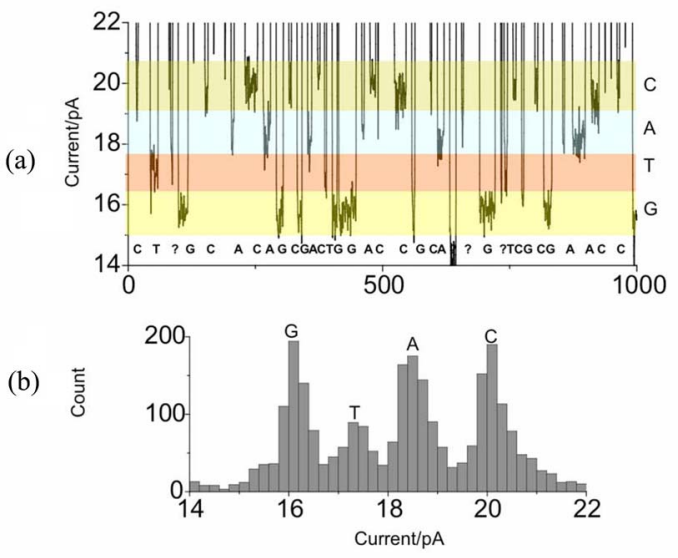

FIG. 18. (Color online) Ionic current through an engineered pore. (a) Current trace with all nucleotides present in solution (time is in milliseconds). (b) Current distributions from the engineered pore. From Astier et al., 2006.

affinity, the pore can interact in a particular way to each of the four bases of DNA. A specific interaction could, for instance, create a different level of pore blockade for each of the bases. In this way, a distinguishable ionic current may be obtainable. The underlying premise was demonstrated by $\mathrm{Gu}$ et al. (1999) with the use of an "adapter" molecule in an $\alpha$-hemolysin pore. The adapter molecule was noncovalently inserted into a pore, and it helped to detect organic molecules that go through the pore by specific binding interactions that change the value of the current blockade and the translocation time.

A very recent article has gone a long way in demonstrating the use of a molecular adapter in sequencing (Astier et al., 2006). These authors have used a mutant $\alpha$-hemolysin pore, see Fig. 17(a), with a positively charged cyclodextrin adapter. The adapter can bind and unbind from the pore (it is not covalently bound), creating a stochastic signal of successive on and off current blockades. The addition of nucleotides changes this stochastic signal, creating additional events with a smaller blockade current when the nucleotide binds to the adapter. These smaller blockade currents are significantly different for the different bases, as shown in Fig. 18. Although the apparatus is chemical, it is really detecting a physical difference between the nucleotides. The difference between the bases results in ionic current distributions with partial overlap, which gives the ability to correctly identify a base to about $93-98 \%$ accuracy. Thus, multiple measurements of each base in a sequence are necessary. Unlike the transverse transport ideas discussed below, multiple measurements may not be a controllable feature of the procedure, and therefore the present technique may require resequencing each strand, or using some sort of oscillating voltage to pull the nucleotide in and out of the adapter to obtain the desired accuracy (Kasianowicz et al., 2002; Di Marzio and Kasianowicz, 2003; Astier et al., 2006).

A full sequencing procedure might employ exonuclease digestion to take a DNA strand and remove a 
base at a time (Astier et al., 2006). The pore itself would have to have the exonuclease attached so that each base is released into the pore and its almost unique blockade signature detected. In addition, in order to actually couple the exonuclease digestion with the pore detection, one has to deal with the fact that the adapter is not covalently attached to the pore and can unbind. Also unknown is whether the nucleotides actually pass through the pore or whether they just bind and unbind from the adapter staying in the cis chamber (where they are added). Further, the exonuclease could create the rate-limiting step. For example, a fast digestion rate of $1000 \mathrm{nt} / \mathrm{s}$ has been observed (Matsuura et al., 2001), which is still much slower than the intrinsic speed of the nanopore experiment, on the order of $10^{6} \mathrm{nt} / \mathrm{s}$. However, the rate will also depend on the binding time scales and the bandwidth of the ionic current measurement.

It is clear from the experiments described in this and earlier sections [as well as results not discussed (Wang et al., 2004)] that the detection capabilities and the ability of ionic current experiments to probe physical processes at the nanoscale are promising. The use of molecular adapters to create a working sequencing method is also promising, but there may need to be additional advancements to increase its intrinsic speed.

\section{Transverse electronic current}

When measuring the ionic current, the size, secondary structure, and base-pore interactions distinguish the bases. One can also measure intrinsic electronic properties of the bases by, for instance, embedding electrodes within a nanopore to measure the transverse current through ss-DNA as it translocates through the pore (Zwolak and Di Ventra, 2005). This method essentially detects the electronic structure of the bases (i.e., the degree of delocalization, shape, and energies of the bases' electronic states) relative to the specific electrodes. A schematic of the idea is shown in Fig. 17(b). We stress that transverse transport is different from the (also interesting and not yet fully solved) problem of longitudinal transport in DNA (Di Ventra and Zwolak, 2004; Endres et al., 2004; Porath et al., 2004). The latter is a complex problem involving a disordered quasi-one-dimensional molecule. Further, measuring transverse currents in other scenarios has been suggested as a way to explore base differences and new physics (Apalkov and Chakraborty, 2005; Maciá, 2005).

Initial calculations on individual nucleotides ideally configured between two nanoscale gold electrodes ${ }^{10}$ [see Fig. 5(b)] indicate that the different bases can give quite different currents under some conditions (Zwolak and

\footnotetext{
${ }^{10}$ Note that the electrodes need not be made of gold. For instance, Golovchenko and co-workers are pursuing the same idea with nanotube electrodes. Nanotube electrodes are likely to be less reactive with the liquid and solid environment and may thus be more stable than metals commonly used for contacts. One may also use the nanotube as part of other detection apparati (Meng et al., 2007).
}

Di Ventra, 2005). However, the different currents are sensitive to the electrode spacing, and also vary greatly if the nucleotides have complete freedom in orientation. For a $1.5 \mathrm{~nm}$ electrode spacing and bases standing upright with respect to electrodes [Fig. 5(b)], the electronic currents for the nucleotides are different by orders of magnitude, except for the nucleotides $\mathrm{G}$ and $\mathrm{C}$, which differ only by about a factor of 2 . The reason for this large difference is the coupling of the bases to the electrodes (Zwolak and Di Ventra, 2005). Due to the different sizes of the bases, as shown in Sec. III, their molecular orbitals have a different spatial extent. The largest base, adenine, thus couples better to the upper electrode. More recently the relation between base orientation or size and conductance was shown by Zhang, Krstić, Zikic, et al. (2006). This is both an advantage, in that it gives added differentiation between the bases, and a disadvantage, because any fluctuations in the orientation of the nucleotide can drastically change the current.

To quantify this, look at the tunneling current through a single energy level $E_{N}$ in the limit of zero bias $V$. For weak coupling, as is the case here, this is given approximately by

$$
I \approx \frac{e^{2}}{\pi \hbar} \frac{\Gamma_{L}\left(E_{F}\right) \Gamma_{R}\left(E_{F}\right)}{\left(E_{F}-E_{N}\right)^{2}} V .
$$

This equation shows that the current is proportional to the two coupling strengths $\Gamma_{L}, \Gamma_{R}$ and inversely proportional to the distance between the energy of the state and the Fermi level $E_{F}$. The different bases have nearly the same energy levels compared to the Fermi level (see Fig. 5), thus this portion is not likely to provide much distinguishability. However, the coupling elements can be very different for the bases because of their different spatial extensions and wave functions (see Sec. III.B).

A preliminary look at the effects of changing orientation showed that the transverse currents are still quite different except for $\mathrm{G}$ and $\mathrm{C}$, thus bolstering the case for sequencing (Zwolak and Di Ventra, 2005). Also, unlike the voltage fluctuation measurements discussed below, the transverse current is minimally affected by nearestneighbor bases because the current is controlled by base-electrode coupling and the energy of the molecular states. These are only slightly influenced by the neighboring bases, so long as the electrode width is on the order of the base spacing ( $\sim 7 \AA$ for extended ss-DNA). The base-electrode coupling is particularly important, and this drops exponentially with the distance of the bases from the electrodes. This is unlike the capacitance method discussed below, in which the Coulomb interaction is long range ${ }^{11}$ and thus nearest neighbors can influence the voltage signal. However, at an electrode spacing of $1.5 \mathrm{~nm}$, the current is already very small, on the

\footnotetext{
${ }^{11}$ At these atomic scales, and inside the pore, screening by the few water molecules present around the bases is not as effective as assuming the macroscopic dielectric constant of water (Lagerqvist et al., 2007c).
} 


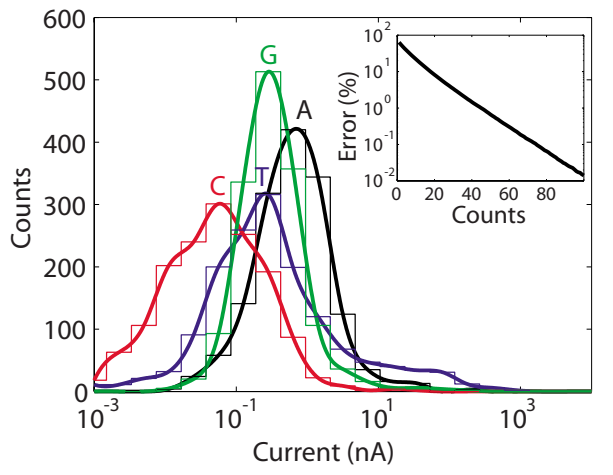

FIG. 19. (Color online) Current distributions for the different nucleotides in a $1.25 \mathrm{~nm}$ electrode spaced pore and at $1 \mathrm{~V}$, with a transverse controlling field. The inset shows the error of misidentifying a base versus the number of independent counts (or measurements). From Lagerqvist et al., 2006.

order of picoamps for the base A. It is thus necessary to go to smaller electrode spacing and also examine the full effect of structural fluctuations.

To obtain a more realistic look at the effect of structural fluctuations, the two authors together with Lagerqvist have performed molecular-dynamics (MD) simulations of ss-DNA being pulled through the pore (Lagerqvist et al., 2006). In the absence of any control on the DNA, the orientation of the bases varies wildly (the bases can be at any orientation, perpendicular or parallel to the electrode surfaces). This causes orders of magnitude fluctuations in the value of the current. Due to these large fluctuations and small values of the current magnitude, it is thus unlikely that sequencing will be possible in the absence of any control on the DNA translocation.

However, MD simulations show that a relatively weak transverse field can orient the nucleotide in the junction with respect to the electrodes in less time (in the hundreds of picoseconds) than it takes the nucleotide to translocate through the junction (Lagerqvist et al., 2006, 2007b). Referring to Fig. 17(b), the condition on the transverse field to be strong enough is $E_{\|} \ll E_{\perp}$. This ensures a slow enough DNA velocity to allow the transverse field to orient the nucleotides while they are facing the electrodes. The transverse field strength can be of the same order of magnitude as that driving the electronic current, but may be provided by an external capacitor around the nanopore device.

When the control is exerted on the nucleotides, one obtains current distributions for the different bases that are sufficiently disjoint (see Fig. 19), so as to allow the bases to be statistically distinguishable. In this case, the statistics can be gathered both intrinsically and extrinsically. First, the strand has some finite velocity, thus allowing for multiple measurements to be gathered as the nucleotide translocates through the junction region (so long as the inverse of the bandwidth of the electrode probes is much smaller than the translocation time of a nucleotide). Second, and more importantly, the finite bandwidth of the probe itself samples over many configurations of the intervening nucleotide.

Overall this leads to a sequencing protocol where one first has to measure the current distributions for each of the nucleotides using a homogeneous strand. These distributions will depend on the particular pore geometry and conditions and thus will be particular to each device. Then one can sequence the DNA by pulling it at a velocity that allows each nucleotide to stay in the electrode region for enough time to collect a current distribution. Then, by comparing with the target distributions, one can determine the sequence (Lagerqvist et al., 2006). To get an idea about the potential speeds achievable with this method, we estimated that a single run through the three billion bases on a single strand of human DNA would take a raw time of about $7 \mathrm{~h}$, without parallelization (Lagerqvist et al., 2006).

There are other issues yet to be resolved, some of which have to be addressed experimentally. For instance, the construction of a suitable nanopore with embedded electrodes is a formidable challenge in the implementation and testing of the method described above. There has been very recent progress, though, in making nanosized pores in between two electrodes (Fischbein and Drndić, 2007). Proof of principle, however, does not require a full working device. The distinguishability of the bases via transverse current measurements can be tested with other methods, such as using a scanning tunneling microscope. This route has been explored by Xu et al. (2007a, 2007b) and also using an STM tip modified with bases (Ohshiro and Umezawa, 2006). The former authors have shown experimentally that the four bases (using isolated nucleotides) provide a distinguishable signal via their HOMO level when measured with a scanning tunneling microscope. Earlier others also examined isolated bases or bases in a strand of DNA with scanning probe techniques (Tao et al., 1993; Tanaka et al., 1999; Hamai et al., 2000; Wang et al., 2001; Tanaka and Kawai, 2003). The idea of using transverse transport to sequence is being pursued experimentally [Ramsey et al. (NHGRI, 2006), Branton and Golovchenko (2007), Fischbein and Drndic (2007), and Lee and Meller (2007)].

There are, of course, drawbacks to the transverse transport approach described. Clearly, if the amount of noise is large, it will make the signals from the bases potentially indistinguishable. There will be $1 / f$ noise and noise provided by ionic fluctuations (discussed below). While one can reduce $1 / f$ noise by operating away from zero frequency, the role of ionic noise is less clear. Also, one may wonder in a real implementation whether clogging of the pore can occur, i.e., whether the small size of the pore and the transverse electric field(s) will cause too much interaction of the DNA with the surface of the pore. This is an issue that has yet to be investigated: its study requires a fully quantum-mechanical treatment of the DNA motion in the pore.

One important and interesting issue, both fundamentally and for the realization of the above approach, is the effect of ionic fluctuations on electronic transport. It has 
importance for many cases of charge transport in soft materials, such as longitudinal transport in DNA (Di Ventra and Zwolak, 2004; Endres et al., 2004; Porath et al., 2004). It would also be interesting to investigate what happens to the tunneling current in the presence of fluctuating energy landscapes created by classical objects (e.g., ions) that cross the electrons' path, when the rate of these fluctuations is comparable to the inverse of the coherence time. An interesting experiment in this direction may be to use different weight counterions to observe the transition from slower to faster fluctuations. The effect of ions on transport is part of the more general problem of understanding the effect of changing a single atom in molecular junctions (Di Ventra et al., 2000; Yang et al., 2003).

Finally, another geometry has been proposed to probe electrical transport perpendicular to the base planes (i.e., with the base plane parallel to the electrode surfaces) (Lee and Meller, 2007). However, there are two challenging issues with this setup. For one, since the transport is now envisioned across the base planes, the electrode spacing has to be very small, $<8 \AA$, to obtain a measurable tunneling current through the bases. On the other hand, this will make it difficult for the ss-DNA to translocate through the pore. From our MD simulations with selected initial conditions (Lagerqvist et al., 2006, 2007b), the smallest pore that will allow DNA to translocate is $10 \AA$ in diameter. In addition, even with an electrode spacing that allows for a measurable current, the differences between the bases in the above planar configuration are not as large in magnitude as in the case in which the bases stand upright with respect to the electrode surfaces [as shown in Fig. 5(b)] (Zwolak and Di Ventra, 2004, 2005). This is due to the fact that the bases have similar HOMO and LUMO charge distributions when viewed perpendicularly to their plane, whereas when standing upright, they have different couplings (see Fig. 6).

\section{Capacitance}

The measurement of voltage fluctuations in a metaloxide-silicon capacitor combined with a nanopore has been proposed as a method to detect and obtain the length of DNA, and potentially to sequence it (Heng, Aksimentiev, Ho, Dimitrov, et al., 2005; Gracheva, Xiong, Aksimentiev, et al., 2006). A schematic of a nanopore capacitor is shown in Fig. 17(c). As each nucleotide passes through the pore, the charge on its backbone can induce a voltage across a capacitor in the longitudinal direction. By measuring these voltage fluctuations, one can effectively count the number of nucleotides. For such a scheme to be used for sequencing, it would also have to be sensitive enough to detect the dipole moment differences of the nucleotides given in Sec. III.

Heng, Aksimentiev, Ho, Dimitrov, et al. (2005) observed voltage signals on the two doped-silicon electrodes as a strand of DNA passes through the pore, the difference of which gives oscillations. The oscillations
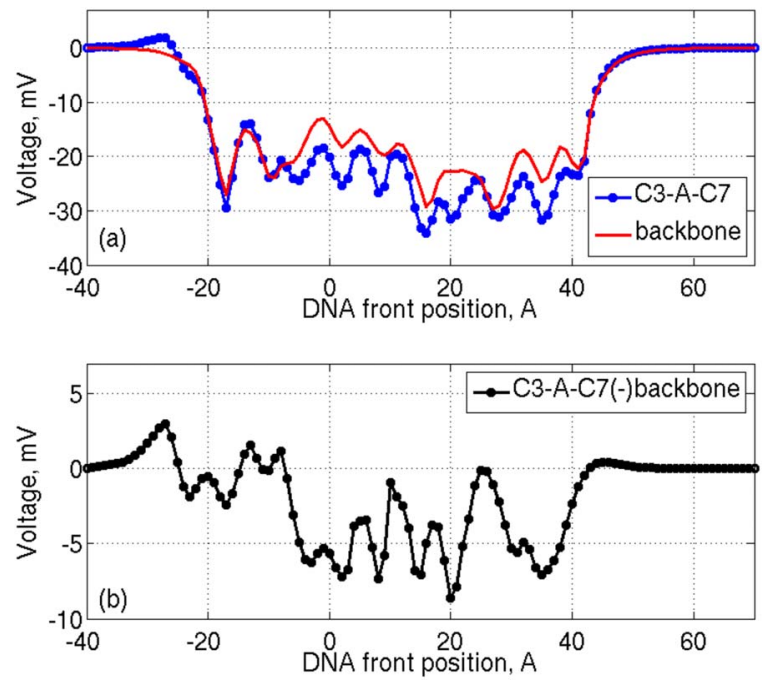

FIG. 20. (Color online) An example voltage signal obtained from MD simulations and electrostatic calculations of an 11 base single strand of DNA translocating through a nanopore capacitor. The dips correspond to the nucleotides. Experimental signals have been obtained of the voltage fluctuations, but without the fine resolution of these simulated signals (Gracheva, Xiong, Aksimentiev, et al., 2006) From Gracheva, Aksimentiev, and Leburton, 2006.

have a magnitude that could be adequate to detect the dipole difference of Adenine and Thymine (Heng, Aksimentiev, Ho, Dimitrov, et al., 2005). However, experimentally these authors were not able to resolve even the charge on the individual nucleotides because of the bandwidth and $R C$ time constant of the probe, and the large size of the pore.

The same group also performed simulations (Gracheva, Aksimintiev, and Leburton, 2006; Gracheva, Xiong, Aksimentiev, et al., 2006) to calculate the expected voltage fluctuations on the capacitor as a ss-DNA is pulled through a $1 \mathrm{~nm}$ radius pore. In these simulations, the DNA strand was not allowed to have conformational fluctuations (similar to the calculation shown in Fig. 1 of Lagerqvist et al., 2006). An example signal obtained from this simulation is shown in Fig. 20. Interestingly, the maximum signal obtained is $\sim 35 \mathrm{mV}$ for the nucleotide, $30 \mathrm{mV}$ for the backbone, and $8 \mathrm{mV}$ for the base. In addition, as a nucleotide moves through the pore, the corresponding voltage signal has been found to be influenced by up to three nearby nucleotides. Even though the bases themselves show different voltage signals, the larger backbone signal seems to dominate and give almost identical signals for the different nucleotides (see Fig. 3 in Gracheva, Aksimentiev, and Leburton, 2006).

Another important factor in the capacitance approach is the dimension of the pore. Estimates suggest it should have about $1 \mathrm{~nm}$ radius in order to obtain an adequate signal (Gracheva, Aksimentiev, and Leburton, 2006; Gracheva, Xiong, Aksimentiev, et al., 2006). The $1 \mathrm{~nm}$ radius of the pore forces the DNA to be stretched as it 
goes through the pore, thus aligning the bases perpendicular to the electrodes (which produces a better signal than if the plane of the bases were parallel to the electrode surfaces) and maximizing their distance from each other [for the sequences in Gracheva, Aksimentiev, and Leburton (2006), the bases are about $7 \AA$ apart, double their distance in ds-DNA or in ss-DNA with strong secondary structure]. Also, the small pore excludes a lot of water, thus reducing screening.

Theoretically, one wonders how much, in principle, the bases can be distinguished via their dipole moments. From Sec. III, the dipole moments of $\mathrm{C}$ and $\mathrm{G}$ isolated bases differ by just 1\% (the differences between $\mathrm{A}$ and $\mathrm{T}$, and those with G, C, are much larger). This, however, ignores the effect of the backbone dipole and the ioncounterion dipole, which are both large. In addition, the large fluctuations of ions at the entrance of the pore may contribute substantially to the voltage fluctuations. Indeed, most of the voltage drop occurs at the location of the (high-resistance) pore. Thus, ions of opposite charge would build up on the two open sides of the pore entrance. When DNA enters the pore, it creates an even higher-resistance pore. As a consequence, there should be a fluctuation in the ionic concentrations near the pore ends, thus creating a temporary voltage fluctuation on the capacitor. The consequence of this effect on sequencing, together with conformational fluctuations of the DNA bases inside the pore, has not been studied yet.

To get some perspective on how meaningful the dipole moments are, consider the dipole moments of other species present in an actual experiment. Water has a gas phase dipole moment of $\sim 1.8 \mathrm{D}$ (Clough et al., 1973). However, in the condensed phase, the dipole moment of water is larger, $\sim 3 \mathrm{D}$ (Silvestrelli and Parrinello, 1999). This is easy to understand: the hydrogen bonding that takes place between water molecules induces more polarization in the electronic distribution, causing an increase in the dipole moment. This is actually true for other species as well: the interactions between the polar solvent and the solute (the PN) will induce larger dipole moments in the solute. More specifically, differences in how water interacts with the different bases could significantly change their dipole moment (this is an open and important question).

In addition, ions present in solution contribute to the dipole moment fluctuations. Consider, for instance, the counterion on the DNA backbone. The values of the deoxyribonucleic acid dipoles given in Table II are for a passivated backbone. The passivation is simply the addition of a hydrogen atom on one of the partially charged oxygen atoms on the phosphate group. This additional hydrogen has a bond length of about $1 \AA$. Therefore, if we consider the effect of a bound counterion, we have to add an additional dipole moment of about $1 e \AA$, because the ion-oxygen bond would be about $1 \AA$ longer. This additional dipole thus contributes 4.8 D. Further, molecular-dynamics simulations indicate that the counterions on the DNA backbone fluctuate quite a lot (Lagerqvist et al., 2007b). Thus, there could be noise larger

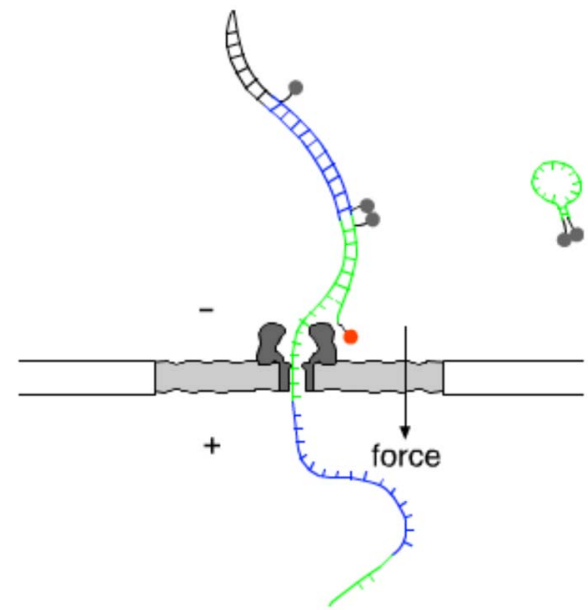

FIG. 21. (Color online) A double strand of DNA with a magnified sequence is pulled through a nanopore. As the strand goes through, it unzips, releasing the fluorescently tagged single strand, which is then read optically. Courtesy of A. Meller.

in magnitude than the nucleotide or base dipole moments themselves, and much larger than the differences between them. However, how this noise interferes with the signal from the bases is not clear, since at these length scales the fluctuations may or may not give a small average noise.

\section{B. Optical detection}

Another proposed method for sequencing relies on optical methods and ds-DNA unzipping via the interaction with a nanopore. The idea is to tear apart "amplified" DNA and then read an optical signal (Lee and Meller, 2007). This process is shown in Fig. 21.

In more detail, one uses a biochemical process to create amplified, or "designer," ds-DNA where each base is represented by a unique $N$-base sequence (with $N \approx 20$ ). These $N$-base sequences are just two blocks of $N / 2$ base homogeneous strands. The $N / 2$ base strands are each labeled with a fluorescent tag on one end and a quenching molecule at the other, such that within the double strand each tag is always paired to a quencher. This prevents fluorescence when the DNA is away from the pore. When the ds-DNA is pulled into, for instance, an $\alpha$-hemolysin pore, the double strand has to unzip into single strands (Sauer-Budge et al., 2003; Mathé et al., 2004, 2006). This pulls the fluorescent tag away from its quencher and allows the tag, and thus the base in the original strand, to be detected via optical means.

Despite the necessity to work with "amplified" DNA, this method has tremendous potential for sequencing, and can be parallelized quite easily by creating several pores in the same device (Lee and Meller, 2007). Preliminary estimates give a raw sequencing rate of $\sim 1-10$ million bases/s for a parallelized nanopore detector. 


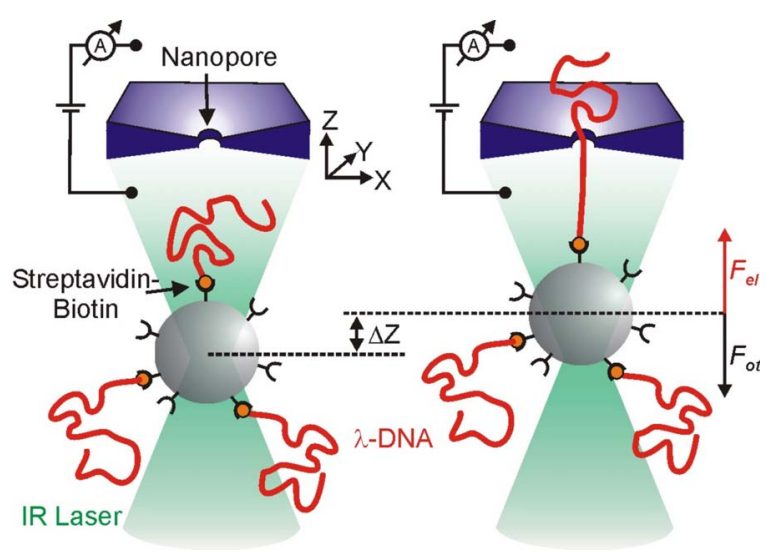

FIG. 22. (Color online) Optical tweezer used in conjunction with a nanopore. From Keyser et al., 2006.

\section{Force detection}

One important technique that may be useful in DNA sequencing and detection, and also single-molecule and nanopore studies, is the use of optical tweezers (Neuman and Block, 2004). An already implemented setup using optical tweezers in conjunction with a nanopore is shown in Fig. 22 (Keyser et al., 2006).

In this experiment, $\lambda$-DNA is attached to polystyrene beads via a streptavidin-biotin interaction [this is noncovalent protein-ligand association, and in this case is very strong (Di Ventra and Zwolak, 2004)]. The dielectric bead can be trapped at the focal point of a tightly focused laser beam (Neuman and Block, 2004). If the bead is pulled away from the focal point, a restoring force is exerted on the bead. For small displacements, this force is linearly proportional to the distance of the bead from the focus, e.g., it resembles a spring obeying Hooke's law. Thus, by measuring the position of the bead from the laser focus, which can be done by measuring the reflected light off the bead, one can determine the net external force on the bead via

$$
F_{\text {ot }}=-k_{\text {trap }} \Delta Z,
$$

where $k_{\text {trap }}$ is the stiffness of the optical trap and $\Delta Z$ is the distance of the bead from the focus of the beam (Keyser et al., 2006).

By bringing the bead close to a nanopore, one can then control the motion of the DNA in the nanopore and measure the forces exerted on it by the pulling voltage. By measuring these forces, Keyser et al. (2006) determined that the effective charge for each nucleotide pair on the double-stranded DNA is about $25 \%$ of its bare value, i.e., $0.5 e$ instead of $2 e$. For $100 \mathrm{mV}$ pulling voltage, this effective charge gives a force on the DNA of $24 \mathrm{pN}$.

Clearly, the concept of coupling the nanopore setup with an optical trap would open up many possibilities to study physics and chemistry of single molecules. One can also imagine many possibilities for using this setup, or more complex setups (for instance, with two optical traps, one on each side of the pore), for sequencing tech- nology and also to help in proof-of-principle prototypes. Keyser et al. (2006) succeeded in slowing the translocation of ds-DNA down by five orders of magnitude compared to its free translocation rate, bringing it down from $\sim 8 \mathrm{~mm} / \mathrm{s}$ to $30 \mathrm{~nm} / \mathrm{s}$. For ionic blockade sequencing, the ability of the optical trap to slow and control the DNA translocation would allow for more definitive experimental measurements of the different ionic blockade currents for the various bases, and may also allow for the stretching of DNA so that secondary structure effects may be reduced or removed.

For transverse current and capacitance measurements, the controlled motion of DNA in the nanopore can also help in a number of ways. For one, it can slow down the DNA so that one can see the gaps between the bases and understand how to count the bases as they go by the pore. If one were to use a more complex setup, with two optical traps on each side of the pore, the pulling voltage could be completely turned off. This would enable the condition $E_{\|} \ll E_{\perp}$ necessary for successful transverse transport sequencing to be satisfied (see Sec. V.A.2). Furthermore, it would reduce ion flow through the pore to a minimum (only Brownian-type motion would be present) and may thus reduce noise from ionic fluctuations for both the transverse current and capacitance.

In addition to the measurement of the effective charge of DNA in solution, optical traps have provided the necessary control and precision force measurements for the study of DNA interacting with RNA polymerase at the single-base level (Abbondanzieri et al., 2005) and even single-molecule sequencing (Greenleaf and Block, 2006).

\section{CONCLUSIONS}

In this Colloquium, we have examined a variety of different proposals for accurate, rapid DNA and RNA sequencing and detection. These methods take physical approaches to detecting single bases in a sequence. Some of the basic ideas have already been implemented experimentally and shown to be useful for detection of strands of DNA and also as probes for "global" properties, such as length or homogeneous sequences. It is, however, still unclear as to whether these results can be extended to true single-molecule sequencing of DNA.

We showed explicitly with ionic blockade and transverse transport that the physical differences of base properties come in the form of differing signal distributions. This will hold true for all other approaches because of the electronic and structural similarity of the bases. Thus, a single, instantaneous measurement signal of a microscopically fluctuating nucleotide cannot be expected to fall within a unique range. This suggests a statistical approach to physical DNA sequencing. In some cases, the statistics may be built into the measurement apparatus via time averaging of the signals, as is the case with finite bandwidth electrical measurements. It is still an open question whether noise, in particular ionic noise, will drown out any signal difference between the 
bases, especially with capacitance or transverse transport measurements. Other noise has to be reduced in the inherently noisy nanopore (or other) environment, and the measurement device has to have sufficient resolution to see the differences in base signals.

Another open issue involves achievable read lengths and sequencing rates. Various lengths of DNA have been pulled through nanopores. For instance, Storm, Storm, Chen, et al. (2005) pulled $\sim 100 \mathrm{kbp}$ ds-DNA through a $10 \mathrm{~nm}$ pore. However, the present authors do not know of a systematic study of possible read lengths of ss-DNA being pulled through nanometer-scale pores. Since the forces on DNA in the pore are small (on the order of tens of piconewtons, much less than the forces necessary to break covalent bonds), practical matters such as entanglement of ss-DNA outside the pore or nonlinear dependence of translocation velocities on DNA length are likely to limit read lengths. Additionally, the translocation rate of each nucleotide through the nanopore has to be smaller than the device bandwidth, allowing each base to stay long enough at its optimal position in the detection apparatus (e.g., in the active detection region, such as between the two electrodes in a transverse transport approach).

Progress in techniques and proof of principle are likely to proceed in part via the use of auxiliary systems. Optical tweezers (Keyser et al., 2006), a nanotube bound to an atomic force microscope (King and Golovchenko, 2005), and other ideas (Krieger, 2006) could give rise to techniques to slow polynucleotide (or molecular) motion in the pore, and also offer the potential to get rid of the ionic solution or electrophoretic pulling. This could be tremendously beneficial in demonstrating proof of concept and/or noise reduction. Further, we alluded to the distinction between chemical and physical processes earlier. Chemical processes change the atomic makeup or structure of a species. They potentially add slow extra steps to a sequencing procedure. However, they can add enhanced distinguishability as well. For instance, one could imagine using the amplification of the optical method together with ionic blockade or transverse transport. Since nanopores can detect differences in homogeneous sequences, it may be possible to detect amplified or designer blocks of nucleotides.

In addition to the technological motivation, there is also a scientific motivation for studying the detection step of a sequencing protocol. The process of directly detecting physical differences between the bases is fundamentally different from the chemical and optical processes currently in use. Thus, not only does it represent a scientific challenge with the goal of low cost and rapid sequencing and detection, it is likely to increase our understanding of biological and other molecules at the atomic scale, and may lead to off-shoot technologies.

In this Colloquium, we have raised several important open questions. Now that fabrication techniques of solid-state pores are maturing, experiments can be tailored to answer these questions more directly by systematically changing pore dimensions, materials, surface properties, and environmental (e.g., ionic) conditions.
With adequately controlled experiments, nanopores could be used to probe inhomogeneities at the nanoscale, such as fine structures in "dielectrics" (e.g., hydration layers) and other interesting physical phenomena.

Because of the widespread technological and scientific interest, it is impossible to predict where this field will go from here. Undoubtedly, ingenious techniques and methodologies will be invented that will extend our abilities to detect and sequence DNA well beyond what has been discussed. Some examples have been mentioned: integration of biological pores with nanoscale probes, use of molecular base discriminators with biological or synthetic pores, and so on. The most promising fundamental research will sort through the many competing effects that exist in these complex systems, and increase our understanding of physical processes at the interface between solids, liquids, and biomolecules down to the nanometer-scale regime. We are confident that a physical approach to DNA detection will yield a wealth of information on physical and chemical processes at the nanoscale, and, hopefully, a rapid and lowcost sequencing technology.

\section{ACKNOWLEDGMENTS}

We thank M. Ramsey for useful discussions, J. Lagerqvist and N. Bushong for a critical reading of the manuscript, and A. Meller for providing information about his suggested sequencing method before publication. M.Z. acknowledges support from the Gordon and Betty Moore Foundation and M.D. acknowledges support from the National Human Genome Research Institute of NIH, Grant No. 2 R01 HG002647-03.

\section{REFERENCES}

Abbondanzieri, E. A., W. J. Greenleaf, J. W. Shaevitz, R. Landick, and S. M. Block, 2005, Nature 438, 460.

Akeson, M., D. Branton, J. J. Kasianowicz, E. Brandin, and D. W. Deamer, 1999, Biophys. J. 77, 3227.

Aksimentiev, A., J. B. Heng, G. Timp, and K. Schulten, 2004, Biophys. J. 87, 2086.

Ambjörnsson, T., S. P. Apell, Z. Konkoli, E. A. D. Marzio, and J. J. Kasianowicz, 2002, J. Chem. Phys. 117, 4063.

Apalkov, V., and T. Chakraborty, 2005, Phys. Rev. B 72, 161102(R).

Arnott, S., R. Chandrasekaran, and A. G. W. Leslie, 1976, J. Mol. Biol. 106, 735.

Ashkenasy, N., J. Sanchez-Quesada, H. Bayley, and M. R. Ghadiri, 2005, Angew. Chem., Int. Ed. 44, 1401.

Astier, Y., O. Braha, and H. Bayley, 2006, J. Am. Chem. Soc. 128, 1705.

Barcilon, V., D. P. Chen, and R. S. Eisenberg, 1992, SIAM J. Appl. Math. 52, 1405.

Bayley, H., 1994, J. Cell. Biochem. 56, 177.

Bayley, H., and P. S. Cremer, 2001, Nature (London) 413, 226.

Bezrukov, S. M., and J. J. Kasianowicz, 1993, Phys. Rev. Lett. 70, 2352.

Bezrukov, S. M., I. Vodyanoy, R. A. Brutyan, and J. J. Kasianowicz, 1996, Macromolecules 29, 8517.

Bezrukov, S. M., I. Vodyanoy, and V. A. Parsegian, 1994, Na- 
ture (London) 370, 279.

Bonthuis, D. J., J. Zhang, B. Hornblower, J. Mathé, B. I. Shklovskii, and A. Meller, 2006, Phys. Rev. Lett. 97, 128104.

Branton, D., and J. A. Golovchenko, 2007, http:// www.mcb.harvard.edu/branton/

Bruskov, V., 1978, Stud. Biophys. 67S, 4344.

Bustamante, J. O., H. Oberleithner, J. A. Hanover, and A. Liepins, 1995, J. Membr. Biol. 146, 253.

Butler, T. Z., J. H. Gundlach, and M. A. Troll, 2006, Biophys. J. 90, 190.

Chan, E. Y., 2005, Mutat Res. 573, 13.

Chang, H., F. Kosari, G. Andreadakis, M. A. Alam, G. Vasmatzis, and R. Bashir, 2004, Nano Lett. 4, 1551.

Chang, H., B. M. Venkatesan, S. M. Iqbal, G. Andreadakis, F. Kosari, G. Vasmatzis, D. Peroulis, and R. Bashir, 2006, Biomed. Microdevices 8, 263.

Chen, D. P., V. Barcilon, and R. S. Eisenberg, 1992, Biophys. J. 61, 1372.

Chen, P., J. Gu, E. Brandin, Y.-R. Kim, Q. Wang, and D. Branton, 2004, Nano Lett. 4, 2293.

Chern, S.-S., A. E. Cárdenas, and R. D. Coalson, 2001, J. Chem. Phys. 115, 7772.

Chuang, J., Y. Kantor, and M. Kardar, 2001, Phys. Rev. E 65, 011802.

Clough, S. A., Y. Beers, G. P. Klein, and L. S. Rothman, 1973, J. Chem. Phys. 59, 2254.

Coalson, R. D., and M. G. Kurnikova, 2005, IEEE Trans. Nanobiosci. 4, 81.

Cui, S. T., 2004, Mol. Phys. 102, 139.

Cui, S. T., 2007, Phys. Rev. Lett. 98, 138101.

Deamer, D. W., and M. Akeson, 2000, Trends Biotechnol. 18, 147.

Deamer, D. W., and D. Branton, 2002, Acc. Chem. Res. 35, 817.

DeGuzman, V. S., C. C. Lee, D. W. Deamer, and W. A. Vercoutere, 2006, Nucleic Acids Res. 34, 6425.

Devoe, H., and I. Tinoco, 1962, J. Mol. Biol. 4, 500.

Dewey, T. G., and D. H. Turner, 1979, Biochemistry 18, 5757.

Di Marzio, E. A., and J. J. Kasianowicz, 2003, J. Chem. Phys. 119, 6378.

Di Ventra, M., S. Evoy, and J. R. Heflin, 2004, Eds., Introduction to Nanoscale Science and Technology (Springer, New York).

Di Ventra, M., S. T. Pantelides, and N. D. Lang, 2000, Phys. Rev. Lett. 84, 979.

Di Ventra, M., and M. Zwolak, 2004, in Encyclopedia of Nanoscience and Nanotechnology, edited by H. Singh-Nalwa (American Scientific Publishers, New York), Vol. 2, p. 475.

Dovichi, N. J., and J. Zhang, 2000, Angew. Chem., Int. Ed. 39, 4463.

Endres, R. G., D. L. Cox, and R. R. P. Singh, 2004, Rev. Mod. Phys. 76, 195.

Fan, R., R. Karnik, M. Yue, D. Li, A. Majumdar, and P. Yang, 2005, Nano Lett. 5, 1633.

Fischbein, M. D., and M. Drndić, 2007, Nano Lett. 7, 1329.

Fologea, D., M. Gershow, B. Ledden, D. S. McNabb, J. A. Golovchenko, and J. Li, 2005, Nano Lett. 5, 1905.

Fologea, D., J. Uplinger, B. Thomas, D. S. McNabb, and J. Li, 2005, Nano Lett. 5, 1734.

Fredlake, C. P., D. G. Hert, E. R. Mardis, and A. E. Barron, 2006, Electrophoresis 27, 3689.

Freier, S. M., K. Hill, T. G. Dewey, L. A. Marky, K. J. Breslauer, and D. H. Turner, 1981, Biochemistry 20, 1419.
Gracheva, M. E., A. Aksimentiev, and J.-P. Leburton, 2006, Nanotechnology 17, 3160.

Gracheva, M. E., A. Xiong, A. Aksimentiev, K. Schulten, G. Timp, and J.-P. Leburton, 2006, Nanotechnology 17, 622.

Greenleaf, W. J., and S. M. Block, 2006, Science 313, 801.

Gu, L.-Q., O. Braha, S. Conlan, S. Cheley, and H. Bayley, 1999, Nature (London) 398, 686.

Hamai, C., H. Tanaka, and T. Kawai, 2000, J. Phys. Chem. B 104, 9894.

Heng, J. B., A. Aksimentiev, C. Ho, V. Dimitrov, T. W. Sorsch, J. F. Miner, W. M. Mansfield, K. Schulten, and G. Timp, 2005, Bell Labs Tech. J. 10, 5.

Heng, J. B., A. Aksimentiev, C. Ho, P. Marks, Y. V. Grinkova, S. Sligar, K. Schulten, and G. Timp, 2005, Nano Lett. 5, 1883.

Heng, J. B., A. Aksimentiev, C. Ho, P. Marks, Y. V. Grinkova, S. Sligar, K. Schulten, and G. Timp, 2006, Biophys. J. 90, 1098.

Heng, J. B., C. Ho, T. Kim, R. Timp, A. Aksimentiev, Y. V. Grinkova, S. Sligar, K. Schulten, and G. Timp, 2004, Biophys. J. 87, 2905.

Henrickson, S. E., M. Misakian, B. Robertson, and J. J. Kasianowicz, 2000, Phys. Rev. Lett. 85, 3057.

Henry, J.-P., J.-F. Chich, D. Goldschmidt, and M. Thieffry, 1989, J. Membr. Biol. 112, 139.

Hille, B., 2001, Ionic Channels of Excitable Membranes, 3rd ed. (Sinauer, Sunderland, MA).

Jackson, J. D., 1998, Classical Electrodynamics, 3rd ed. (Wiley, New York).

Jenkins, J., D. Sengupta, and S. Sundaram, 2005, Lect. Notes Comput. Sci. 3516, 309.

JGI, 2004, http://www.jgi.doe.gov/education/how/

Kamenev, A., J. Zhang, A. Larkin, and B. Shklovskii, 2006, Physica A 359, 129.

Kasianowicz, J. J., and S. M. Bezrukov, 1995, Biophys. J. 69, 94.

Kasianowicz, J. J., E. Brandin, D. Branton, and D. W. Deamer, 1996, Proc. Natl. Acad. Sci. U.S.A. 93, 13770.

Kasianowicz, J. J., S. E. Henrickson, M. Misakian, H. H. Weetall, B. Robertson, and V. M. Stanford, 2002, in Structure and Dynamics of Confined Polymers, edited by J. J. Kasianowicz, M. Kellermayer, and D. W. Deamer (Kluwer, Dordrecht), p. 141.

Kasianowicz, J. J., S. E. Henrickson, H. H. Weetall, and B. Robertson, 2001, Anal. Chem. 73, 2268.

Keyser, U. F., B. N. Koeleman, S. V. Dorp, D. Krapf, R. M. M. Smeets, S. G. Lemay, N. H. Dekker, and C. Dekker, 2006, Nat. Phys. 2, 473.

King, G. M., and J. A. Golovchenko, 2005, Phys. Rev. Lett. 95, 216103.

Kong, C. Y., and M. Muthukumar, 2002, Electrophoresis 23, 2697.

Krieger, K., 2006, Anal. Chem. 78, 3486.

Kulakows, I., M. Geller, B. Lesyng, and K. L. Wierzcho, 1974, Biochim. Biophys. Acta 361, 119.

Lagerqvist, J., M. Zwolak, and M. Di Ventra, 2006, Nano Lett. 6, 779 .

Lagerqvist, J., M. Zwolak, and M. Di Ventra, 2007a, Biophys. J. 93, 2384

Lagerqvist, J., M. Zwolak, and M. Di Ventra, 2007b, Phys. Rev. E 76, 013901.

Lagerqvist, J., M. Zwolak, and M. Di Ventra, 2007c, unpublished.

Lander, E. S., et al., 2001, Nature (London) 409, 860921. 
Lee, J. W., and A. Meller, 2007, in Perspectives in Bioanalysis, edited by K. Mitchelson (Elsevier, Amsterdam).

Li, J., M. Gershow, D. Stein, E. Brandin, and J. A. Golovchenko, 2003, Nat. Mater. 2, 611.

Li, J., D. Stein, C. McMullan, D. Branton, M. J. Aziz, and J. A. Golovchenko, 2001, Nature (London) 412, 166.

Lindsay, S. M., et al., 2007, http://green.la.asu.edu

Loebl, H. C., R. Randel, S. P. Goodwin, and C. C. Matthai, 2003, Phys. Rev. E 67, 041913.

Lubensky, D. K., and D. R. Nelson, 1999, Biophys. J. 77, 1824. Luo, K., T. Ala-Nissila, and S.-C. Ying, 2006, J. Chem. Phys. 124, 034714.

Maciá, E., 2005, Rev. Adv. Mater. Sci. 10, 166.

Mathé, J., A. Aksimentiev, D. R. Nelson, K. Schulten, and A. Meller, 2005, Proc. Natl. Acad. Sci. U.S.A. 102, 12377.

Mathé, J., A. Arinstein, Y. Rabin, and A. Meller, 2006, Europhys. Lett. 73, 128.

Mathé, J., H. V. V. Viasnoff, Y. Rabin, and A. Meller, 2004, Biophys. J. 87, 3205.

Matsuura, S.-i., J. K. K. Hirano, H. Y. K. Takashima, S. Katsura, and A. Mizuno, 2001, Nucleic Acids Res. 29, e79.

Matysiak, S., A. Montesi, M. Pasquali, A. B. Kolomeisky, and C. Clementi, 2006, Phys. Rev. Lett. 96, 118103.

Meller, A., 2003, J. Phys.: Condens. Matter 15, R581.

Meller, A., L. Nivon, E. Brandin, J. Golovchenko, and D. Branton, 2000, Proc. Natl. Acad. Sci. U.S.A. 97, 1079.

Meller, A., L. Nivon, and D. Branton, 2001, Phys. Rev. Lett. 86, 3435 .

Meng, S., P. Maragakis, C. Papaloukas, and E. Kaxiras, 2007, Nano Lett. 7, 45.

Muthukumar, M., 1999, J. Chem. Phys. 111, 10371.

Muthukumar, M., 2001, Phys. Rev. Lett. 86, 3188.

Muthukumar, M., and C. Y. Kong, 2006, Proc. Natl. Acad. Sci. U.S.A. 103, 5273.

Nakane, J. J., M. Akeson, and A. Marziali, 2003, J. Phys.: Condens. Matter 15, R1365.

Neuman, K., and S. Block, 2004, Rev. Sci. Instrum. 75, 2787.

NHGRI, 2006, http://www.genome.gov/19518500, see also www.genome.gov/12513210

Ohshiro, T., and Y. Umezawa, 2006, Proc. Natl. Acad. Sci. U.S.A. 103, 10.

Porath, D., G. Cuniberti, and R. D. Felice, 2004, Top. Curr. Chem. 237, 183.

Saenger, W., 1988, Principles of Nucleic Acid Structure (Springer, New York).

Sanger, F., S. Nicklen, and A. R. Coulson, 1977, Proc. Natl. Acad. Sci. U.S.A. 74, 5463.

Sauer-Budge, A. F., J. A. Nyamwanda, D. K. Lubensky, and D. Branton, 2003, Phys. Rev. Lett. 90, 238101.

Schuss, Z., B. Nadler, and R. S. Eisenberg, 2001, Phys. Rev. E 64, 036116.

Searle, M. S., and D. H. Williams, 1993, Nucleic Acids Res. 21, 2051.

Silvestrelli, P. L., and M. Parrinello, 1999, Phys. Rev. Lett. 82,
3308.

Siwy, Z., L. Trofin, P. Kohli, L. A. Baker, C. Trautmann, and C. R. Martin, 2005, J. Am. Chem. Soc. 127, 5000.

Slonkina, E., and A. B. Kolomeisky, 2003, J. Chem. Phys. 118, 7112.

Song, L., M. R. Hobaugh, C. Shustak, S. Cheley, H. Bayley, and J. E. Gouaux, 1996, Science 274, 1859.

Storm, A. J., J. H. Chen, X. S. Ling, H. Zandbergen, and C. Dekker, 2003, Nat. Mater. 2, 537540.

Storm, A. J., J. H. Chen, H. W. Zandbergen, and C. Dekker, 2005, Phys. Rev. E 71, 051903.

Storm, A. J., C. Storm, J. Chen, H. Zandbergen, J.-F. Joanny, and C. Dekker, 2005, Nano Lett. 5, 1193.

Sung, W., and P. J. Park, 1996, Phys. Rev. Lett. 77, 783.

Tanaka, H., C. Hamai, T. Kanno, and T. Kawai, 1999, Surf. Sci. 432, L611.

Tanaka, H., and T. Kawai, 2003, Surf. Sci. 539, L531.

Tao, N. J., J. A. DeRose, and S. M. Lindsay, 1993, J. Phys. Chem. 97, 910.

Teber, S., 2005, J. Stat. Mech.: Theory Exp. P07001 (2005).

Tsai, Y.-S., and C.-M. Chen, 2007, J. Chem. Phys. 126, 144910.

Venter, J. C., et al., 2001, Science 291, 1304.

Vercoutere, W., S. Winters-Hilt, H. Olsen, D. Deamer, D. Haussler, and M. Akeson, 2001, Nat. Biotechnol. 19, 248.

Vercoutere, W. A., S. Winters-Hilt, V. S. DeGuzman, D. Deamer, S. E. Ridino, J. T. Rodgers, H. E. Olsen, A. Marziali, and M. Akeson, 2003, Nucleic Acids Res. 31, 1311.

Vesnaver, G., and K. J. Breslauer, 1991, Proc. Natl. Acad. Sci. U.S.A. 88, 3569.

Wang, H., J. E. Dunning, A. P.-H. Huang, J. A. Nyamwanda, and D. Branton, 2004, Proc. Natl. Acad. Sci. U.S.A. 101, 13472 .

Wang, H., Z. Tang, Z. Li, and E. Wang, 2001, Surf. Sci. 480, L389.

Wanunu, M., and A. Meller, 2007, Nano Lett. 7, 1580.

Weber, H. P., and B. M. Craven, 1990, Acta Crystallogr., Sect. B: Struct. Sci. 46, 532.

Winters-Hilt, S., W. Vercoutere, V. S. DeGuzman, D. Deamer, M. Akeson, and D. Haussler, 2003, Biophys. J. 84, 967.

Xu, M., R. G. Endres, and Y. Arakawa, 2007a, in Nano Science and Technology, edited by K. v. Klitzing (Springer-Verlag, Heidelberg).

Xu, M., R. G. Endres, and Y. Arakawa, 2007b, Small 3, 1539.

Yang, Z., N. D. Lang, and M. Di Ventra, 2003, Appl. Phys. Lett. 82, 1938.

Zhang, J., A. Kamenev, and B. I. Shklovskii, 2005, Phys. Rev. Lett. 95, 148101.

Zhang, J., A. Kamenev, and B. I. Shklovskii, 2006, Phys. Rev. E 73, 051205.

Zhang, J., and B. I. Shklovskii, 2007, Phys. Rev. E 75, 021906. Zhang, X.-G., P. S. Krstić, R. Zikic, J. C. Wells, and M. Fuentes-Cabrera, 2006, Biophys. J. 91, L04.

Zwolak, M., and M. Di Ventra, 2004, unpublished.

Zwolak, M., and M. Di Ventra, 2005, Nano Lett. 5, 421. 